\title{
Relativistic and pseudorelativistic formulation of nonlinear envelope equations with spatiotemporal dispersion. II. Saturable systems
}

\author{
J. M. Christian,* G. S. McDonald, M. J. Lundie, and A. Kotsampaseris \\ Joule Physics Laboratory, School of Computing, Science and Engineering, \\ University of Salford, Greater Manchester M5 4WT, United Kingdom
}

(Dated: July 26, 2018)

\begin{abstract}
We consider an envelope equation with space-time symmetry for describing scalar waves in systems with spatiotemporal dispersion and a generic saturable nonlinearity. Exact bright and gray solitons are derived by direct integration methods and coordinate transformations, with the results for cubicquintic systems [see companion article-submitted to Phys. Rev. A] recovered in the limit of weak saturation. Classic predictions from a nonlinear-Schrödinger formulation of the propagation problem are shown to emerge asymptotically as subsets of the more general spatiotemporal solutions. The robustness of the new solitons against perturbations to the local pulse shape is then tested by deploying integral stability criteria, symmetry principles, and numerical analysis.
\end{abstract}

PACS numbers: 42.65.-k, 42.65.Fs, 42.65.Tg, 42.65.Wi, 05.45.Yv

Keywords: bright solitons, dark solitons, Kerr effect, spatial dispersion, waveguide optics

\section{INTRODUCTION}

Solitons and solitary-wave phenomena are elementary excitations, pervasive throughout Nature, that play a key role in modern understandings of nonlinear systems [1]. Regardless of the physical context - optical or fluidic, mechanical or electromagnetic, biological or chemical, classical or quantum - the emergence of these robust particlelike wave states generally requires just two basic ingredients: linear dispersion and nonlinearity [2]. Models supporting envelope solitons are historically rooted in universal equations such as the nonlinear-Schrödinger (NLS) type, and in the simplest case they describe waveforms that are localized in time $t$ and travelling through space $z$. Longitudinal modulations to a rapidly-varying harmonic signal are typically assumed to take place on a scalelength that is much greater than the carrier wavelength [the slowly-varying envelope approximation (SVEA)].

The SVEA is often a valid starting point for analyses [3] and the undoubted success of NLS-based models in predicting experimentally-observed phenomena (e.g., in optics [4]) has allowed them to become linchpins of conventional pulse theory [5]. The SVEA is usually complemented by a Galilean transformation, where for convenience one boosts from the laboratory frame to a localtime frame moving at the group velocity $v_{g}$, as defined by coordinates $z_{\mathrm{loc}} \equiv z$ and $t_{\mathrm{loc}} \equiv t-z / v_{g}$. It is instructive, however, to pose questions about the properties of wavepackets beyond the more traditional level of description. What are the governing equations? Do they have exact non-trivial solutions? Can invariance laws and conserved quantities be identified? What are the implications for stability? Can predictions be reconciled with the SVEA? etc. We have made some progress in answering these fundamental questions by proposing

* Corresponding author: j.christian@salford.ac.uk a spatiotemporal formulation based on generic envelope equations that respect space-time symmetry [6].

The spatiotemporal nomenclature has different interpretations so it is helpful to begin by clarifying the terminology used throughout (the situation is akin to nonparaxial when discussing beams in spatial optics [7]). It is often associated with the phenomenon of wave-like "bullets" where the interplay between nonlinearity, groupvelocity dispersion (GVD) and diffraction can sustain (at least in the short term) wave self-confinement in transverse and longitudinal spatial directions in addition to being localized in time [8]. One also encounters the term in Ginzburg-Landau contexts when analyzing pattern emergence, bifurcations, and chaotic dynamics under the combined action of gain and loss [9]. Here, we take it to mean the simultaneous presence of both spatial and temporal dispersion in any wave-based system [6].

Mathematically, our quite general conception of spatiotemporal dispersion lies with the appearance of both $\partial^{2} / \partial z^{2}$ and $\partial^{2} / \partial t^{2}$ operators in the envelope equation (which may or may not also involve formal diffractive considerations). It has application in the field of nonlinear optics, supplementing the widely-known SVEAbased models of light propagation [3-5] with a more complete geometrical theory [6]. It also has practical uses in terms of modelling condensed-matter effects in some special classes of semiconductor (e.g., ZnCdSe/ZnSe superlattices). Bianacalana and Creatore [10] have previously demonstrated that material spatial dispersion due to photon-exciton coupling cannot be captured by SVEAtype frameworks, and that retaining full generality of $\partial^{2} / \partial z^{2}$ facilitates an adequate description.

To date, the classic cubic [11] and cubic-quintic (see companion article [12]) nonlinearities have been investigated in some detail with particular emphasis placed on deriving exact bright and dark solitons. One of the most self-evident and intriguing results is that our spatiotemporal formulation has strong analogues with special relativity [13]. The invariance laws are either rela- 
tivistic or pseudorelativistic (depending on the interplay between spatial and temporal dispersion), and as a consequence (inverse) velocities add geometrically through a rule that is reminiscent of the familiar Lorentz form. Perhaps most striking is the recovery of SVEA-type results. They emerge asymptotically through a multiplelimit procedure that is entirely equivalent to the way Newtonian physics reappears from special relativity at low speeds. For completeness, there remains one final class of system nonlinearity that needs to be addressed.

Saturation is a universal phenomenon that tends to inhibit unphysical run-away effects. In optics, for example, it is a principal feature of Maxwell-Bloch cavity models involving the continuous interaction between a circulating light beam and a system comprising atoms with two discrete energy levels [14]. Such a system tends to exhibit saturation in its medium polarization and population inversion when electric-dipole coupling is subjected to highintensity pumping. In cavityless configurations, such as a dispersive waveguide, one might intuitively expect the nonlinear dielectric response (e.g., permittivity or refractive index) of the core material [15] to support a maximum allowable change before approaching the threshold for optical damage. However, the standard cubic (i.e., Kerr) $[3,16]$ and cubic-quintic $[17,18]$ approximations permit arbitrarily-large contributions to self-interaction effects. These models are based on simple scalar power series (or, more rigorously, on tensor expansions of the medium polarization and the introduction of nonlinear susceptibilities $[16,19])$ that are truncated after a finite number of terms (e.g., on the basis of order-of-magnitude considerations). In contrast, the plateauing that characterizes saturation (i.e., where a physical property can sustain no further self-induced variation) does not always lend itself well to polynomial-type representations that, inevitably, fail at sufficiently high light intensity.

The saturating responses of many optical materials have been measured over the years. Such experimental studies have included some semiconductor-doped glasses (e.g., CdSSe and Schott OG 550 glass) [20], ion-doped crystals (e.g., $\mathrm{GdAlO}_{3}: \mathrm{Cr}^{3+}$ ) [21], bio-optical media [22], $\pi$-conjugated polymers [23], and various photorefractive crystals (e.g., $\mathrm{LiNbO}_{3}$ and SBN) [24].

While several trial functions are available for describing a saturable refractive index [25-27], all of which share similar qualitative features, our principal interest lies with that proposed by Wood, Evans, and Kenan [28]. Their model appears to be unique in that it allows the corresponding governing equations to be integrated exactly - for instance, families of transverse guided modes in dielectric planar waveguides were obtained by solving the Helmholtz equation and enforcing continuity conditions at the boundary between substrates. More pertinently, exact bright [29] and dark [30] solitons derived by Krolikowski and Luther-Davies are also known within the context of Schrödinger equations. It is these latter solution classes that we seek to generalize here and, in so-doing, more fully develop our un- derstanding of waves in space-time-symmetric systems by accounting for saturation effects. Known spatiotemporal cubic-quintic [12] and cubic [11] solitons are then expected to emerge as special cases, forming a natural hierarchy of solutions.

The layout of this paper is as follows. In Sec. II, we explore the spatiotemporal envelope equation in the context of the saturable nonlinearity and formulate the intensityphase quadrature problem. Exact bright and dark solitons are derived in Secs. III and IV, respectively, with coordinate transformations deployed in Sec. V to obtain more general results (including a discussion of nondegenerate bistability characteristics). Rigorous asymptotic analyses are detailed in Sec. VI with regards various important physical limits, with predictions about soliton stability made and tested against full simulations in Sec. VII. We conclude, in Sec. VIII, with some remarks about connections to other potential research avenues.

\section{SPATIOTEMPORAL MODEL}

\section{A. Envelope equation}

We consider the governing equation for a dimensionless envelope $u$ that is given by

$$
\begin{aligned}
\kappa \frac{\partial^{2} u}{\partial \zeta^{2}}+i\left(\frac{\partial u}{\partial \zeta}\right. & \left.+\alpha \frac{\partial u}{\partial \tau}\right)+\frac{s}{2} \frac{\partial^{2} u}{\partial \tau^{2}} \\
& +\frac{1}{2} \frac{2+|u|^{2} / \rho_{\text {sat }}}{\left(1+|u|^{2} / \rho_{\text {sat }}\right)^{2}}|u|^{2} u=0
\end{aligned}
$$

where $\zeta$ and $\tau$ are normalized space and time coordinates, respectively, as measured in the laboratory frame. The linear part of the wave operator in Eq. (1) is generic (in terms of its dispersive contributions and space-time symmetry) while the nonlinear part is homogeneous and parametrized by $\rho_{\text {sat }}$ (the normalized saturation intensity). We assume a set of units such that GVD is controlled by $s= \pm 1$ ( +1 for anomalous, -1 for normal) and the parameter $\alpha$ is a ratio of group velocities. Spatial dispersion is determined by $\kappa \ll \mathcal{O}(1)$, and it is taken to be positive without loss of generality. An example scaling is given in Appendix A.

To clarify, here we define relativistic and pseudorelativistic scenarios as being those characterized by $s=-1$ and $s=+1$, respectively, whereupon the transformation laws of Eq. (1) correspond to skews and rotations in the $(\tau, \zeta)$ plane $[11]$.

In the context of Eq. (1), the SVEA is embodied by the inequality $\kappa\left|\partial^{2} u / \partial \zeta^{2}\right| \ll|\partial u / \partial \zeta|$. Throughout, we purposely avoid that regime (both analytically and computationally) until considering the position of conventional model predictions within the wider soliton hierarchy. Neglecting the operator $\kappa \partial^{2} / \partial \zeta^{2}$ will then be shown as synonymous with a simultaneous multiple limit in the algebra of spatiotemporal solutions. Moreover, we have found 
that $\kappa \partial^{2} / \partial \zeta^{2}$ can be accommodated in many exact analyses and thus it does not need to be treated perturbatively (e.g., through order-of-magnitude considerations [31]).

\section{B. General quadrature equations}

Solutions to Eq. (1) are sought that have a general form described by the Madelung-type ansatz

$$
u(\tau, \zeta)=\rho^{1 / 2}(\tau, \zeta) \exp [i \psi(\tau, \zeta)],
$$

where $\rho$ and $\psi$ are real functions determining the intensity and (total) phase, respectively, of $u$. Substituting Eq. (2a) into Eq. (1) and collecting the real and imaginary parts yields the following pair of coupled equations:

$$
\begin{gathered}
\frac{2}{\rho}\left(\frac{\partial^{2} \rho}{\partial \tau^{2}}+2 s \kappa \frac{\partial^{2} \rho}{\partial \zeta^{2}}\right)-\frac{1}{\rho^{2}}\left[\left(\frac{\partial \rho}{\partial \tau}\right)^{2}+2 s \kappa\left(\frac{\partial \rho}{\partial \zeta}\right)^{2}\right] \\
-4\left[\left(\frac{\partial \psi}{\partial \tau}\right)^{2}+2 s \kappa\left(\frac{\partial \psi}{\partial \zeta}\right)^{2}\right]-8 s\left(\frac{\partial \psi}{\partial \zeta}+\alpha \frac{\partial \psi}{\partial \tau}\right) \\
+8 s \frac{2+\rho / \rho_{\text {sat }}}{\left(1+\rho / \rho_{\text {sat }}\right)^{2}}\left(\frac{\rho}{2}\right)=0 \\
\rho\left(\frac{\partial^{2} \psi}{\partial \tau^{2}}+2 s \kappa \frac{\partial^{2} \psi}{\partial \zeta^{2}}\right)+\left(\frac{\partial \psi}{\partial \tau} \frac{\partial \rho}{\partial \tau}+2 s \kappa \frac{\partial \psi}{\partial \zeta} \frac{\partial \rho}{\partial \zeta}\right) \\
+s\left(\frac{\partial \rho}{\partial \zeta}+\alpha \frac{\partial \rho}{\partial \tau}\right)=0
\end{gathered}
$$

(neglecting all the $\kappa$-dependent terms leads to the wellknown fluid-type equations [32] for $\rho$ and $\psi$ in the corresponding SVEA-type model). One can now eliminate the carrier-wave part of the solution by setting

$$
\psi(\tau, \zeta)=\Psi(\tau, \zeta)+K \zeta-\frac{\zeta}{2 \kappa} .
$$

Here, $\Psi$ denotes the phase distribution for the solitary excitation, $K$ is the propagation constant, and the final factor describes the rapidly-oscillating component inherent to the solutions of models that are second-order in $\zeta$ [11]. Quadrature equations (2b) and (2c) may then be written as

$$
\begin{gathered}
\frac{2}{\rho}\left(\frac{\partial^{2} \rho}{\partial \tau^{2}}+2 s \kappa \frac{\partial^{2} \rho}{\partial \zeta^{2}}\right)-\frac{1}{\rho^{2}}\left[\left(\frac{\partial \rho}{\partial \tau}\right)^{2}+2 s \kappa\left(\frac{\partial \rho}{\partial \zeta}\right)^{2}\right] \\
-4\left[\left(\frac{\partial \Psi}{\partial \tau}\right)^{2}+2 s \kappa\left(\frac{\partial \Psi}{\partial \zeta}\right)^{2}\right] \\
-8 s\left(\alpha \frac{\partial \Psi}{\partial \tau}+2 \kappa K \frac{\partial \Psi}{\partial \zeta}\right) \\
-8 s\left[\beta-\frac{2+\rho / \rho_{\text {sat }}}{\left(1+\rho / \rho_{\text {sat }}\right)^{2}}\left(\frac{\rho}{2}\right)\right]=0
\end{gathered}
$$

and

$$
\begin{aligned}
& \rho\left(\frac{\partial^{2} \Psi}{\partial \tau^{2}}+2 s \kappa \frac{\partial^{2} \Psi}{\partial \zeta^{2}}\right)+\left(\frac{\partial \Psi}{\partial \tau} \frac{\partial \rho}{\partial \tau}+2 s \kappa \frac{\partial \Psi}{\partial \zeta} \frac{\partial \rho}{\partial \zeta}\right) \\
& \quad+s\left(\alpha \frac{\partial \rho}{\partial \tau}+2 \kappa K \frac{\partial \rho}{\partial \zeta}\right)=0 .
\end{aligned}
$$

Here, we have defined the dispersion relation by identifying $\kappa K^{2}-1 / 4 \kappa \equiv \beta$ so that

$$
K= \pm \frac{1}{2 \kappa} \sqrt{1+4 \kappa \beta}
$$

and where the \pm sign denotes propagation in the forward $(+)$ or backward $(-)$ longitudinal direction.

\section{Space-time coordinate transformation \& symmetry reduction}

Inspection of Eqs. (4a) and (4b) demonstrates that there is a symmetry between space and time derivatives that does not appear in the conventional approach to pulse modelling. Such symmetry can be exploited by introducing the lumped space-time coordinate $\xi \equiv \xi(\tau, \zeta)$, effectively a single new independent variable, where

$$
\xi(\tau, \zeta) \equiv \frac{\tau-V_{0} \zeta}{\sqrt{1+2 s \kappa V_{0}^{2}}}
$$

and $V_{0}$ is a constant intrinsic velocity that parametrizes the transformation. By implementing change-ofvariables (5) through the replacement of partial differential operators $\partial / \partial \tau$ and $\partial / \partial \zeta$ and combinations thereofsee Ref. [12] for details - we can use Eqs. (4a) and (4b) to write down a pair of coupled ordinary differential equations for the $\rho$ and $\Psi$ quadratures in terms of $\xi$ :

$$
\begin{gathered}
\frac{d}{d \rho}\left[\frac{1}{\rho}\left(\frac{d \rho}{d \xi}\right)^{2}\right]-4\left(\frac{d \Psi}{d \xi}\right)^{2}-8 s\left(\frac{\alpha-2 \kappa K V_{0}}{\sqrt{1+2 s \kappa V_{0}^{2}}}\right) \frac{d \Psi}{d \xi} \\
-8 s\left[\beta-\frac{2+\rho / \rho_{\text {sat }}}{\left(1+\rho / \rho_{\text {sat }}\right)^{2}}\left(\frac{\rho}{2}\right)\right]=0 \\
\rho \frac{d^{2} \Psi}{d \xi^{2}}+\frac{d \Psi}{d \xi} \frac{d \rho}{d \xi}+s\left(\frac{\alpha-2 \kappa K V_{0}}{\sqrt{1+2 s \kappa V_{0}^{2}}}\right) \frac{d \rho}{d \xi}=0
\end{gathered}
$$

To find particular (e.g., soliton) solutions, Eqs. (6a) and (6b) must be supplemented by appropriate boundary conditions on $\rho(\xi)$ and $\Psi(\xi)$.

\section{BRIGHT SOLITON PULSES}

One expects bright solitons to exist in the case of anomalous GVD (where $s=+1$ ). In the following analysis, the 'b' subscript is introduced to denote bright solitons through $\rho(\xi) \rightarrow \rho_{\mathrm{b}}(\xi), \Psi(\xi) \rightarrow \Psi_{\mathrm{b}}(\xi), \beta \rightarrow \beta_{\mathrm{b}}, K \rightarrow$ $K_{\mathrm{b}}$, and the intrinsic velocity is flagged by $V_{0} \rightarrow V_{0 \mathrm{~b}}$. 


\section{A. Intensity quadrature}

To obtain the quadrature equations for bright solitons, we look for particular solutions where $\Psi_{\mathrm{b}}=0$ so that there is no phase change across the temporal width of the pulse. Equation (6a) becomes

$$
\frac{d}{d \rho_{\mathrm{b}}}\left[\frac{1}{\rho_{\mathrm{b}}}\left(\frac{d \rho_{\mathrm{b}}}{d \xi}\right)^{2}\right]=8\left[\beta_{\mathrm{b}}-\frac{2+\rho_{\mathrm{b}} / \rho_{\mathrm{sat}}}{\left(1+\rho_{\mathrm{b}} / \rho_{\mathrm{sat}}\right)^{2}}\left(\frac{\rho_{\mathrm{b}}}{2}\right)\right],
$$

and direct integration with respect to $\rho_{\mathrm{b}}$ leads to

$$
\left(\frac{d \rho_{\mathrm{b}}}{d \xi}\right)^{2}=8 \beta_{\mathrm{b}} \rho_{\mathrm{b}}^{2}-4 \rho_{\mathrm{sat}}^{2}\left(\frac{\rho_{\mathrm{b}}^{2}}{\rho_{\mathrm{sat}}}+\frac{\rho_{\mathrm{b}}}{1+\rho_{\mathrm{b}} / \rho_{\mathrm{sat}}}\right)+c_{2 \mathrm{~b}} \rho_{\mathrm{b}},
$$

where $c_{2 \mathrm{~b}}$ is a constant to be determined from the solution boundary conditions. As $\xi \rightarrow \pm \infty$, the intensity profile must decay to zero sufficiently rapidly so that $\rho_{\mathrm{b}} \rightarrow 0$ as $\left(d \rho_{\mathrm{b}} / d \xi\right)^{2} \rightarrow 0$. Applying these limits to Eq. (7b) shows that $c_{2 \mathrm{~b}}=4 \rho_{\text {sat }}^{2}$ (note that, in the cubicquintic regime [12], the corresponding constant of integration vanishes). Similarly, when $\xi \rightarrow 0$ the intensity tends to its peak value, denoted by $\rho_{0}$, so that

$$
\beta_{\mathrm{b}}=\frac{1}{1+\rho_{0} / \rho_{\mathrm{sat}}}\left(\frac{\rho_{0}}{2}\right) \text {. }
$$

The bright soliton propagation constant $K_{\mathrm{b}}$, given in Eq. (4c), is then fully determined.

By combining the algebraic results for $c_{2 \mathrm{~b}}$ and $\beta_{\mathrm{b}}$, the right-hand side of Eq. (7b) can be factorized to yield the more compact structure

$$
\left(\frac{d \rho_{\mathrm{b}}}{d \xi}\right)^{2}=4 \rho_{\mathrm{b}}^{2} \frac{\left(\rho_{0}-\rho_{\mathrm{b}}\right)}{\left(1+\rho_{0} / \rho_{\mathrm{sat}}\right)\left(1+\rho_{\mathrm{b}} / \rho_{\mathrm{sat}}\right)} .
$$

A second integration uncovers an implicit result describing the spatiotemporal pulse intensity profile which, for ease of comparison, we express in a form similar to that introduced by Krolikowski and Luther-Davies [29]:

$$
\begin{gathered}
\tan ^{-1}\left(\eta_{\mathrm{b}}\right)+\frac{1}{2}\left(\frac{\rho_{\mathrm{sat}}}{\rho_{0}}\right)^{1 / 2} \ln \left[\frac{\left(\rho_{0} / \rho_{\mathrm{sat}}\right)^{1 / 2}+\eta_{\mathrm{b}}}{\left(\rho_{0} / \rho_{\mathrm{sat}}\right)^{1 / 2}-\eta_{\mathrm{b}}}\right] \\
=\frac{\rho_{\mathrm{sat}}^{1 / 2}}{\left(1+\rho_{0} / \rho_{\mathrm{sat}}\right)^{1 / 2}} \xi,
\end{gathered}
$$

where

$$
\eta_{\mathrm{b}}\left[\rho_{\mathrm{b}}\right] \equiv\left(\frac{\rho_{0}-\rho_{\mathrm{b}}}{\rho_{\mathrm{sat}}+\rho_{\mathrm{b}}}\right)^{1 / 2}
$$

is a positive real parameter and $\eta_{\mathrm{b}}(\xi) \equiv \eta_{\mathrm{b}}\left[\rho_{\mathrm{b}}(\xi)\right]$. Equations $(9 \mathrm{a})$ and $(9 \mathrm{~b})$ can then be solved numerically to obtain $\rho_{\mathrm{b}}(\xi)$ (see Fig. 1). One finds that at fixed $\rho_{0}$, pulse widths tend to decrease with increasing $\rho_{\text {sat }}$.

One of the most notable aspects of the preceding analysis is that a closed-form prediction for the soliton profile

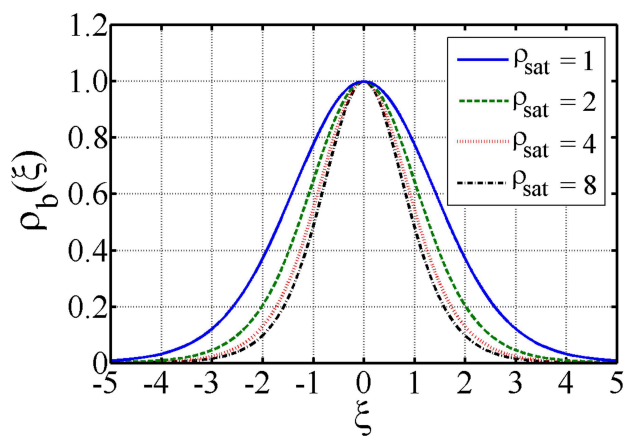

FIG. 1: (color online) Bright soliton intensity distributions obtained by solving Eq. (9a) for $\rho_{0}=1.0$. These profiles are universal since they are insensitive to variations in $\kappa, \alpha$, and

$V_{0 \mathrm{~b}}$. The dilation (pulse broadening) effect appears for anomalous GVD $(s=+1)$ when considering these curves as functions of $\tau$ rather than of $\xi[12]$.

can be obtained. It is surprising, then, that while the complicated nonlinearity of Wood et al. [28] yields exact results, much simpler variants do not [29]. Indeed, Gatz and Herrmann have shown that NLS-type bright solitons of two other saturable refractive-index models (involving two-level atoms [25] and double-doping [27]) may be obtained only in the form of integral equations (to be solved iteratively) or through direct numerical solution of the quadrature problem. Similar intractability is also present in spatiotemporal regimes since the system of ordinary differential equations often turns out to be the same (e.g., see Appendix B) but where $\xi$ and the local-time coordinate are effectively interchangeable.

\section{B. Intrinsic velocity}

One must now ensure that Eq. (6b) is also rigorously satisfied where, for $\Psi_{\mathrm{b}}=0$, it assumes the simple form

$$
\left(\alpha-2 \kappa K_{\mathrm{b}} V_{\mathrm{ob}}\right) \frac{d \rho_{\mathrm{b}}}{d \xi}=0 .
$$

In order for Eq. (10a) to hold true for arbitrary gradients $d \rho_{\mathrm{b}} / d \xi$, one must have $\alpha-2 \kappa K_{\mathrm{b}} V_{0 \mathrm{~b}}=0$, or equivalently $V_{0 \mathrm{~b}}=\alpha / 2 \kappa K_{\mathrm{b}}$. Hence,

$$
V_{0 \mathrm{~b}}= \pm \frac{\alpha}{\sqrt{1+4 \kappa \beta_{\mathrm{b}}}}
$$

and where the structure of $V_{0 \mathrm{~b}}$ here is identical to that for other known bright spatiotemporal solitons [11, 12]. Such a structure arises from the space-time symmetry inherent to the linear part of the wave operator in Eq. (1). In contrast, the connection between $\beta_{\mathrm{b}}$ and $\rho_{0}$ [c.f., Eq. (7c)] depends upon the details of the system nonlinearity.

Since the pulse travels (in dimensional units) at a velocity that is proportional to $1 / V_{0 \mathrm{~b}}$ (a quantity defined in dimensionless units), one can immediately see that spatiotemporal solitons must be assigned speeds that have 
a weak dependence on intensity. This type of effect is not typically encountered in NLS-type models, but it is nonetheless present in other universal nonlinear wave equations. For instance, the canonical Korteweg de-Vries model with third-order linear dispersion and a quadratic self-steepening term predicts a fundamental $\mathrm{sech}^{2}$-shaped soliton with an amplitude that is directly proportional to its speed (an essential component for describing wavebreaking phenomena) [1].

\section{DARK SOLITON PULSES}

One expects dark solitons to exist in the case of normal GVD (where $s=-1$ ). Since the localized component of $u$ resides as a dip travelling across a background continuous-wave $(\mathrm{cw})$ solution, we set $\rho(\xi) \rightarrow \rho_{\mathrm{d}}(\xi)$, $\Psi(\xi) \rightarrow \Psi_{\mathrm{d}}(\xi)$, and $V_{0} \rightarrow V_{0 \mathrm{~d}}$ where the 'd' subscript denotes dark solitons. The remaining two parameters are labelled as $\beta \rightarrow \beta_{\mathrm{cw}}$ and $K \rightarrow K_{\mathrm{cw}}$ with reference to the cw component.

\section{A. Continuous-wave solutions}

The cw solutions of Eq. (1) have a uniform intensity $\rho_{0}$ and may be assigned a finite frequency shift $\Omega$ so that

$$
u_{\mathrm{cw}}(\tau, \zeta)=\rho_{0}^{1 / 2} \exp \left[i\left(-\Omega \tau+K_{\mathrm{cw}} \zeta\right)\right] \exp \left(-i \frac{\zeta}{2 \kappa}\right)
$$

and $\left|u_{\mathrm{cw}}\right|^{2} \equiv \rho_{0}$. Substitution of ansatz (11a) in Eq. (1) yields the dispersion relation

$$
\kappa K_{\mathrm{cw}}^{2}-\Omega\left(\alpha-s \frac{\Omega}{2}\right)-\frac{1}{4 \kappa}=\beta_{\mathrm{cw}},
$$

which is parametrized by

$$
\beta_{\mathrm{cw}} \equiv \frac{2+\rho_{0} / \rho_{\mathrm{sat}}}{\left(1+\rho_{0} / \rho_{\mathrm{sat}}\right)^{2}}\left(\frac{\rho_{0}}{2}\right)
$$

Note that dispersion relation (11b) is either elliptic (when $s=+1$ ) or hyperbolic (when $s=-1$ ) and thus always has two branches corresponding to propagation in the forward and backward longitudinal senses [11].

The stability of the cw background, which is essential for the dark soliton, can be addressed through linear analysis (see Appendix $\mathrm{C}$ for details with regard to generic nonlinearity functionals). Solution (11a) is perturbed by a small-amplitude periodic spatiotemporal modulation with temporal frequency $\Omega_{\mathrm{p}}$. Any long-wavelength disturbance then grows whenever

$$
\frac{\Omega_{\mathrm{p}}^{2}}{2}-2 s \frac{\rho_{0}}{\left(1+\rho_{0} / \rho_{\text {sat }}\right)^{3}}<0 .
$$

Instability is clearly present in the anomalous-GVD regime, but it disappears entirely for normal GVD since inequality (12) can never be satisfied for any $\rho_{0} \geq 0$ (details are given in Appendix C). Hence, the cw background of spatiotemporal dark solitons in saturable systems have the desired modulational stability properties.

\section{B. Quadrature equations}

Having established the MI characteristics of the cw solutions to Eq. (1), we now turn our attention to its dark solitons. The quadrature equations are

$$
\begin{aligned}
\frac{d}{d \rho_{\mathrm{d}}}\left[\frac{1}{\rho_{\mathrm{d}}}\left(\frac{d \rho_{\mathrm{d}}}{d \xi}\right)^{2}\right]= & 4\left(\frac{d \Psi_{\mathrm{d}}}{d \xi}\right)^{2} \\
& -8\left(\frac{\alpha-2 \kappa K_{\mathrm{cw}} V_{0}}{\sqrt{1-2 \kappa V_{0 \mathrm{~d}}^{2}}}\right) \frac{d \Psi_{\mathrm{d}}}{d \xi} \\
& -8\left[\beta_{\mathrm{cw}}-\frac{2+\rho_{\mathrm{d}} / \rho_{\mathrm{sat}}}{\left(1+\rho_{\mathrm{d}} / \rho_{\mathrm{sat}}\right)^{2}}\left(\frac{\rho_{\mathrm{d}}}{2}\right)\right]
\end{aligned}
$$

and

$$
\frac{d}{d \xi}\left[\left(\frac{d \Psi_{\mathrm{d}}}{d \xi}-\frac{\alpha-2 \kappa K_{\mathrm{cw}} V_{0 \mathrm{~d}}}{\sqrt{1-2 \kappa V_{0}^{2}}}\right) \rho_{\mathrm{d}}\right]=0
$$

Integration of Eq. (13b) leads to a result for the phase derivative,

$$
\frac{d \Psi_{\mathrm{d}}}{d \xi}=\left(\frac{\alpha-2 \kappa K_{\mathrm{cw}} V_{0 \mathrm{~d}}}{\sqrt{1-2 \kappa V_{0 \mathrm{~d}}^{2}}}\right)+\frac{c_{1 \mathrm{~d}}}{\rho_{\mathrm{d}}}
$$

where $c_{1 \mathrm{~d}}$ is a constant to be determined by considering the behaviour of $\Psi_{\mathrm{d}}$ as $\xi \rightarrow \pm \infty$. Eliminating $d \Psi_{\mathrm{d}} / d \xi$ terms from Eq. (13a) using Eq. (14a) leaves an equation solely for the intensity quadrature:

$$
\begin{aligned}
\frac{d}{d \rho_{\mathrm{d}}}\left[\frac{1}{\rho_{\mathrm{d}}}\left(\frac{d \rho_{\mathrm{d}}}{d \xi}\right)^{2}\right]= & 4 \frac{c_{1 \mathrm{~d}}^{2}}{\rho_{\mathrm{d}}^{2}}-4\left(\frac{\alpha-2 \kappa K_{\mathrm{cw}} V_{0 \mathrm{~d}}}{\sqrt{1-2 \kappa V_{0 \mathrm{~d}}^{2}}}\right)^{2} \\
& -8\left[\beta_{\mathrm{cw}}-\frac{2+\rho_{\mathrm{d}} / \rho_{\mathrm{sat}}}{\left(1+\rho_{\mathrm{d}} / \rho_{\mathrm{sat}}\right)^{2}}\left(\frac{\rho_{\mathrm{d}}}{2}\right)\right] .
\end{aligned}
$$

Equations (14a) and (14b) are the dark-soliton analogues of Eqs. (7a) and (10a) describing bright solitons. They can be solved to provide an exact solution. 


\section{Intensity quadrature}

Integration of Eq. (14b) with respect to $\rho_{\mathrm{d}}$ gives

$$
\begin{aligned}
\left(\frac{d \rho_{\mathrm{d}}}{d \xi}\right)^{2}= & 4 \rho_{\mathrm{sat}}^{2}\left(\frac{\rho_{\mathrm{d}}^{2}}{\rho_{\mathrm{sat}}}+\frac{\rho_{\mathrm{d}}}{1+\rho_{\mathrm{d}} / \rho_{\mathrm{sat}}}\right) \\
& -4\left[2 \beta_{\mathrm{cw}}+\left(\frac{\alpha-2 \kappa K_{\mathrm{cw}} V_{0 \mathrm{~d}}}{\sqrt{1-2 \kappa V_{0 \mathrm{~d}}^{2}}}\right)^{2}\right] \rho_{\mathrm{d}}^{2} \\
& +c_{2 \mathrm{~d}} \rho_{\mathrm{d}}-4 c_{1 \mathrm{~d}}^{2}
\end{aligned}
$$

By writing $\left(d \rho_{\mathrm{d}} / d \xi\right)^{2}=4 D\left(\rho_{\mathrm{d}}-\rho_{1}\right)\left(\rho_{0}-\rho_{\mathrm{d}}\right)^{2} /(1+$ $\left.\rho_{\mathrm{d}} / \rho_{\text {sat }}\right)$, one may expand the numerator and compare the coefficients of $\rho_{\mathrm{d}}^{n}$ with $n=3,2,1$, and 0 . The result is a system of four auxiliary algebraic equations:

$$
\begin{aligned}
D & \equiv 1+\frac{c_{3 \mathrm{~d}}}{\rho_{\mathrm{sat}}} \\
-D\left(2 \rho_{0}+\rho_{1}\right) & \equiv \frac{c_{2 \mathrm{~d}}}{4 \rho_{\mathrm{sat}}}+\rho_{\mathrm{sat}}+c_{3 \mathrm{~d}}, \\
D\left(\rho_{0}^{2}+2 \rho_{0} \rho_{1}\right) & \equiv \frac{c_{2 \mathrm{~d}}}{4}-\frac{c_{1 \mathrm{~d}}^{2}}{\rho_{\mathrm{sat}}}+\rho_{\mathrm{sat}}^{2} \\
D \rho_{0}^{2} \rho_{1} & \equiv c_{1 \mathrm{~d}}^{2},
\end{aligned}
$$

and where we have introduced the parametrization

$$
c_{3 \mathrm{~d}} \equiv-\left[2 \beta_{\mathrm{cw}}+\left(\frac{\alpha-2 \kappa K_{\mathrm{cw}} V_{0 \mathrm{~d}}}{\sqrt{1-2 \kappa V_{0 \mathrm{~d}}^{2}}}\right)^{2}\right]
$$

for compactness. Equations (16a)-(16d) can be solved sequentially to yield algebraic expressions for constants $D, c_{2 \mathrm{~d}}$ and $c_{1 \mathrm{~d}}$ :

$$
\begin{aligned}
D & =\left(1+\frac{\rho_{0}}{\rho_{\text {sat }}}\right)^{-2}\left(1+\frac{\rho_{1}}{\rho_{\text {sat }}}\right)^{-1}, \\
c_{2 \mathrm{~d}} & =4 D\left[\rho_{0}^{2}\left(1+\frac{\rho_{1}}{\rho_{\text {sat }}}\right)+2 \rho_{0} \rho_{1}\right]-4 \rho_{\text {sat }}^{2}, \\
c_{1 \mathrm{~d}}^{2} & =\frac{\rho_{0}^{2} \rho_{1}}{\left(1+\rho_{1} / \rho_{\text {sat }}\right)\left(1+\rho_{0} / \rho_{\text {sat }}\right)^{2}},
\end{aligned}
$$

though only $D$ and $c_{1 \mathrm{~d}}$ are used from here onwards. It then follows that Eq. (15) may be replaced by the factorized form

$$
\left(\frac{d \rho_{\mathrm{d}}}{d \xi}\right)^{2}=4 \frac{\left(\rho_{0}-\rho_{\mathrm{d}}\right)^{2}\left(\rho_{\mathrm{d}}-\rho_{1}\right)}{\left(1+\rho_{0} / \rho_{\mathrm{sat}}\right)^{2}\left(1+\rho_{1} / \rho_{\mathrm{sat}}\right)\left(1+\rho_{\mathrm{d}} / \rho_{\mathrm{sat}}\right)} .
$$

In the domain where $\xi>0$, the intensity gradient $d \rho_{\mathrm{d}} / d \xi$ must be positive. With these signs in mind, one can perform an integration of Eq. (18) to arrive at an implicit spatiotemporal solution for $\rho_{\mathrm{d}}(\xi)$ that we express in the form first presented by Krolikowski and Luther-Davies [30]:

$$
\begin{aligned}
& \left(\frac{\rho_{0}+\rho_{\mathrm{sat}}}{\rho_{0}-\rho_{1}}\right)^{1 / 2} \tanh ^{-1}\left[\left(\frac{\rho_{0}+\rho_{\mathrm{sat}}}{\rho_{0}-\rho_{1}}\right)^{1 / 2} \eta_{\mathrm{d}}\right] \\
& \quad-\tanh ^{-1}\left(\eta_{\mathrm{d}}\right)=\frac{\rho_{\mathrm{sat}}^{1 / 2}}{\left(1+\rho_{0} / \rho_{\mathrm{sat}}\right)\left(1+\rho_{1} / \rho_{\mathrm{sat}}\right)^{1 / 2}} \xi
\end{aligned}
$$

where

$$
\eta_{\mathrm{d}}\left[\rho_{\mathrm{d}}\right] \equiv\left(\frac{\rho_{\mathrm{d}}-\rho_{1}}{\rho_{\mathrm{d}}+\rho_{\mathrm{sat}}}\right)^{1 / 2}
$$

is a positive real parameter and $\eta_{\mathrm{d}}(\xi) \equiv \eta_{\mathrm{d}}\left[\rho_{\mathrm{d}}(\xi)\right]$. Equations (19a) and (19b) describe the exact (although implicit) dark soliton intensity profile, and they may be solved numerically to yield $\rho_{\mathrm{d}}(\xi)$ (see Fig. 2). For fixed $\rho_{0}$ and $\rho_{1}$, the width of the pulse increases as $\rho_{\text {sat }}$ decreases. The physical nature of this inverse relationship follows directly from the nonlinearity-dispersion balance required for stationary states to exist (any reduction in self-phase modulation must be accompanied by a commensurate weakening of linear spreading) [1, 16].

\section{Intrinsic velocity}

An expression for the intrinsic velocity $V_{0 \mathrm{~d}}$ can be derived by respecting the asymptotic behaviour of the solution. As $\xi \rightarrow \pm \infty$, the intensity tends towards its $\mathrm{cw}$ value $\rho_{0}$ and the phase gradient $d \Psi_{\mathrm{d}} / d \xi \rightarrow 0$ tends to zero. From Eq. (14a), it thus follows that

$$
\frac{\alpha-2 \kappa K_{\mathrm{cw}} V_{0 \mathrm{~d}}}{\sqrt{1-2 \kappa V_{0 \mathrm{~d}}^{2}}}=-\frac{c_{1 \mathrm{~d}}}{\rho_{0}} .
$$

Agreement between Eqs. (16a) and (16b) demands that $\rho_{\text {sat }}(1-B)-2 \beta_{\mathrm{cw}}=B \rho_{1}$ (a result that can be confirmed algebraically). It is then straightforward to show that $V_{0 d}$ satisfies the quadratic equation

$$
\begin{aligned}
& {\left[\left(2 \kappa K_{\mathrm{cw}}\right)^{2}+2 \kappa V_{0 \mathrm{~d} \mathrm{loc}}^{2}\right] V_{0 \mathrm{~d}}^{2}} \\
& \quad-2 \alpha\left(2 \kappa K_{\mathrm{cw}}\right) V_{0 \mathrm{~d}}+\left(\alpha^{2}-V_{0 \mathrm{~d} \mathrm{loc}}^{2}\right)=0
\end{aligned}
$$

where we have defined $V_{0 \mathrm{~d} \text { loc }} \equiv c_{1 \mathrm{~d}} / \rho_{0}$. Analysis of the two roots of Eq. (20b) must be performed with the two branches of $K_{\mathrm{cw}}$ in mind [c.f., Eq. (11b)]. Under these conditions, and being careful to choose the correct sign for forward- $(+)$ and backward-travelling $(-)$ solitons, one finds that the intrinsic velocity is given by 


$$
V_{0 \mathrm{~d}}(F)= \pm \frac{V_{0 \mathrm{~d} \text { loc }}(F)\left\{1+2 \kappa \rho_{0}\left(1+\rho_{0} / \rho_{\mathrm{sat}}\right)^{-2}\left[2+\rho_{0} / \rho_{\mathrm{sat}}+F^{2}\left(1+F^{2} \rho_{0} / \rho_{\mathrm{sat}}\right)^{-1}\right]-2 \kappa \alpha^{2}\right\}^{1 / 2}+\alpha \sqrt{1+4 \kappa \beta_{\mathrm{cw}}}}{1+2 \kappa \rho_{0}\left(1+\rho_{0} / \rho_{\mathrm{sat}}\right)^{-2}\left[2+\rho_{0} / \rho_{\mathrm{sat}}+F^{2}\left(1+F^{2} \rho_{0} / \rho_{\mathrm{sat}}\right)^{-1}\right]}
$$

where the physical interpretation of

$$
V_{0 \mathrm{~d} l o c}(F) \equiv \frac{\rho_{0}^{1 / 2} F}{\left(1+\rho_{0} / \rho_{\mathrm{sat}}\right)\left(1+F^{2} \rho_{0} / \rho_{\mathrm{sat}}\right)^{1 / 2}}
$$

will become clear in Sec. VIB. Here, the dark soliton contrast parameter $F^{2} \equiv \rho_{1} / \rho_{0}$, with $A^{2}+F^{2}=1$ and $0<F^{2} \leq 1$, has been introduced for universal notation [5, 11]. Inspection of Eq. (21b) also shows that, unlike for the competing cubic-quintic nonlinearity [12], there is generally no upper limit on the allowed value of $\rho_{0}$.

\section{E. Phase quadrature}

By combining Eqs. (14a) and (20a), it can be shown that the soliton phase $\Phi_{\mathrm{d}}(\xi)$ is given by the integral

$$
\Psi_{\mathrm{d}}(\xi)=\left(\frac{c_{1 \mathrm{~d}}}{\rho_{0}}\right) \int d \xi\left(\frac{\rho_{0}-\rho_{\mathrm{d}}}{\rho_{\mathrm{d}}}\right)+\Psi_{\mathrm{d} 0},
$$

where $\Psi_{\mathrm{d} 0}$ is a constant that can be set to zero without loss of generality. The intensity profile is not known explicitly as a function of $\xi$, but by deploying Eq. (18) an exact expression for the phase can be obtained wherein the integration is over $\rho_{\mathrm{d}}$. In the domain $\xi \geq 0$,

$$
\begin{aligned}
\Psi_{\mathrm{d}}\left[\eta_{\mathrm{d}}\right]= & \tan ^{-1}\left[\left(\frac{1}{F}\right)\left(\frac{\rho_{\text {sat }}}{\rho_{0}}\right)^{1 / 2} \eta_{\mathrm{d}}\right] \\
& +F\left(\frac{\rho_{0}}{\rho_{\text {sat }}}\right)^{1 / 2} \tanh ^{-1}\left(\eta_{\mathrm{d}}\right)
\end{aligned}
$$

and we note that $\Psi_{\mathrm{d}}(-\xi)=-\Psi_{\mathrm{d}}(\xi)$. As expected, the parity of the dark soliton is determined by the sign of $F$, so under the change $F \rightarrow-F$ the phase profile is reversed in the $\xi$ coordinate. The phase change across the dark soliton, $\Delta \Psi_{\mathrm{d}} \equiv \Psi_{\mathrm{d}}(+\infty)-\Psi_{\mathrm{d}}(-\infty)$, can be expressed as

$$
\begin{aligned}
\Delta \Psi_{\mathrm{d}}= & -2 \tan ^{-1}\left[\left(\frac{F}{A}\right)\left(1+\frac{\rho_{0}}{\rho_{\mathrm{sat}}}\right)^{1 / 2}\right] \\
+ & 2 F\left(\frac{\rho_{0}}{\rho_{\mathrm{sat}}}\right)^{1 / 2} \\
& \times \tanh ^{-1}\left[A\left(\frac{\rho_{0}}{\rho_{\mathrm{sat}}}\right)^{1 / 2}\left(1+\frac{\rho_{0}}{\rho_{\mathrm{sat}}}\right)^{-1 / 2}\right] .
\end{aligned}
$$

Under some conditions, $\Delta \Psi_{\mathrm{d}}$ can be greater than $\pi$ radians (regimes in which the solution has been designated "darker than black" by Krolikowski et al. [33]).

\section{MORE GENERAL SOLUTIONS}

\section{A. Frequency-velocity relations}

The covariance of Eq. (1) under rotations or skews of the space-time coordinate axes means that one may always observe the waves it describes from any arbitrarilydefined frame of reference. This degree of freedom permits one to construct more general solution families that involve a finite frequency shift, denoted here by $\Omega$, so that now $u_{\mathrm{b}} \propto \exp (i \Omega \tau)$ and $u_{\mathrm{d}} \propto \exp (-i \Omega \tau)$.

Following the methods established in Refs. [11] and [12], one may relate $\Omega$ to a transformation parameter $V_{\mathrm{b}}$ or $V_{\mathrm{d}}$ characterizing the coordinate change according to

$$
V_{\mathrm{b}, \mathrm{d}}(\Omega)=\frac{(\Omega+\alpha) \sqrt{1+4 \kappa \beta_{\mathrm{b}, \mathrm{cw}}-4 s \kappa \Omega(\alpha+\Omega / 2)}-\alpha \sqrt{1+4 \kappa \beta_{\mathrm{b}, \mathrm{cw}}}}{1+4 \kappa \beta_{\mathrm{b}, \mathrm{cw}}-2 s \kappa(\Omega+\alpha)^{2}},
$$

and where $V_{\mathrm{b}, \mathrm{d}}$ has a status analogous to the transverse velocity typically introduced into the analysis of obliquely-propagating nonlinear beams [7].

In the spatiotemporal formulation, velocities can be shown to combine geometrically (akin to those in special relativity [13], as determined by the Lorentz rule) rather than additively (as in Galilean relativity). Hence, the net velocity parameters $W_{\mathrm{b}, \mathrm{d}}$ defined in the laboratory frame, being the resultant of the intrinsic and transverse contributions, can be obtained through a rule that is either pseudorelativistic (when $s=+1$ and the geometry is thus Euclidean) or relativistic (when $s=-1$ and the geometry is instead Riemannian) in nature [6]:

$$
W_{\mathrm{b}, \mathrm{d}}=\frac{V_{0 \mathrm{~b}, 0 \mathrm{~d}}+V_{\mathrm{b}, \mathrm{d}}}{1-2 s \kappa V_{0 \mathrm{~b}, 0 \mathrm{~d}} V_{\mathrm{b}, \mathrm{d}}},
$$

After some algebra, combining Eqs. (10b), (24) and (25a) one can show that the net velocity of bright solitons has 
the compact form

$$
W_{\mathrm{b}}= \pm \frac{\alpha+\Omega}{\sqrt{1+4 \kappa \beta_{\mathrm{b}}-4 \kappa \Omega\left(\alpha+\frac{1}{2} \Omega\right)}}
$$

where the \pm sign denoted forward- $(+)$ and backwardtravelling waves $(-)$. An analogous expression for $W_{\mathrm{d}}$ is somewhat cumbersome and does not exhibit such algebraic simplification.

Having introduced a transverse velocity, the lumped space-time variable $\xi$ describing the intensity and phase quadratures [c.f. Eqs. (9a) and (19a)] must be replaced with its transformed counterpart,

$$
\Theta_{\mathrm{b}, \mathrm{d}}(\tau, \zeta) \equiv \frac{\tau \mp W_{\mathrm{b}, \mathrm{d}} \zeta}{\sqrt{1+2 s \kappa W_{\mathrm{b}, \mathrm{d}}^{2}}},
$$

where $\Theta_{\mathrm{b}}$ is associated with $s=+1$ and bright solution, while $\Theta_{\mathrm{d}}$ is selected when considering $s=-1$ and dark solutions. For notational convenience, the \pm sign inherent to $W_{\mathrm{b}, \mathrm{d}}$ [e.g., see Eq. (25b)] has been absorbed into the $\Theta_{b, d}$ variable so as the net velocity parameter is now always a positive quantity. The frequency-shifted bright soliton of Eq. (1) is now given by

$$
\begin{aligned}
& u_{\mathrm{b}}(\tau, \zeta)=\rho_{\mathrm{b}}^{1 / 2}(\tau, \zeta) \\
& \times \exp \left[i \Omega \tau \pm i \sqrt{1+4 \kappa \beta_{\mathrm{b}}-4 \kappa \Omega\left(\alpha+\frac{\Omega}{2}\right)} \frac{\zeta}{2 \kappa}\right] \\
& \quad \times \exp \left(-i \frac{\zeta}{2 \kappa}\right)
\end{aligned}
$$

where $\rho_{\mathrm{b}}(\tau, \zeta)$ is obtained from

$$
\begin{aligned}
\tan ^{-1}\left[\eta_{\mathrm{b}}(\tau, \zeta)\right] \\
+\frac{1}{2}\left(\frac{\rho_{\mathrm{sat}}}{\rho_{0}}\right)^{1 / 2} \ln \left[\frac{\left(\rho_{0} / \rho_{\mathrm{sat}}\right)^{1 / 2}+\eta_{\mathrm{b}}(\tau, \zeta)}{\left(\rho_{0} / \rho_{\mathrm{sat}}\right)^{1 / 2}-\eta_{\mathrm{b}}(\tau, \zeta)}\right] \\
\quad=\frac{\rho_{\mathrm{sat}}^{1 / 2}}{\left(1+\rho_{0} / \rho_{\mathrm{sat}}\right)^{1 / 2}} \Theta_{\mathrm{b}}(\tau, \zeta) .
\end{aligned}
$$

and $\eta_{\mathrm{b}}$ retains its formal definition from Eq. (9b). Similarly, the frequency-shifted dark soliton is

$$
\begin{aligned}
u_{\mathrm{d}}(\tau, \zeta)= & \rho_{\mathrm{d}}^{1 / 2}(\tau, \zeta) \exp \left[i\left\{\tan ^{-1}\left[\left(\frac{1}{F}\right)\left(\frac{\rho_{\mathrm{sat}}}{\rho_{0}}\right)^{1 / 2} \eta_{\mathrm{d}}(\tau, \zeta)\right]+F\left(\frac{\rho_{0}}{\rho_{\mathrm{sat}}}\right)^{1 / 2} \tanh ^{-1}\left[\eta_{\mathrm{d}}(\tau, \zeta)\right]\right\}\right] \\
& \times \exp \left[-i \Omega \tau \pm i \sqrt{1+4 \kappa \beta_{\mathrm{cw}}+4 \kappa \Omega\left(\alpha+\frac{\Omega}{2}\right)} \frac{\zeta}{2 \kappa}\right] \exp \left(-i \frac{\zeta}{2 \kappa}\right)
\end{aligned}
$$

where

$$
\begin{aligned}
\left(\frac{\rho_{\mathrm{sat}}}{\rho_{0}}\right)^{1 / 2} \frac{\left(1+\rho_{0} / \rho_{\mathrm{sat}}\right)^{1 / 2}}{A} \tanh ^{-1}\left[\left(\frac{\rho_{\mathrm{sat}}}{\rho_{0}}\right)^{1 / 2} \frac{\left(1+\rho_{0} / \rho_{\mathrm{sat}}\right)^{1 / 2}}{A} \eta_{\mathrm{d}}(\tau, \zeta)\right] & \rho_{\mathrm{sat}}^{1 / 2} \\
-\tanh ^{-1}\left[\eta_{\mathrm{d}}(\tau, \zeta)\right] & =\frac{\left(1+\rho_{0} / \rho_{\mathrm{sat}}\right)\left[1+\left(1-A^{2}\right) \rho_{0} / \rho_{\mathrm{sat}}\right]^{1 / 2}}{} \Theta_{\mathrm{d}}(\tau, \zeta)
\end{aligned}
$$

and $\eta_{\mathrm{d}}$ still has the formal definition from Eq. (19b). Both solutions have now been parametrized by the ratio $\rho_{0} / \rho_{\text {sat }}$, which faciliates a more straightforward asymptotic analysis of waveforms in the weak-saturation limit $\left[\right.$ defined to be $\left.\rho_{0} / \rho_{\text {sat }} \ll \mathcal{O}(1)\right]$.

\section{B. Non-degenerate bistability}

As with cubic-quintic systems $[12,34,35]$, it is possible to sweep across the solution continua for the saturable system and identify pairs of non-degenerate bistable pulses. For the case of bright solitons, there emerges a range of values for $\rho_{\text {sat }}$ within which two solitary pulses may have different peak intensities while sharing the same full-width-half maximum (FWHM) [29, 30]. Such a bistable characteristic is different from that proposed by Kaplan [36] describing degenerate solitons, where the integrated intensity may become a multi-valued function of the propagation constant. It is also distinct from the familiar $\mathcal{S}$-shaped response curve from external feedback in driven nonlinear cavities [14] (e.g., as described by the roots of a cubic equation modelling the steady states of a plane wave experiencing interferomic mistuning, periodic pumping, and coupling-mirror losses).

Non-degenerate bistable bright solitons with a FWHM of $2 \nu \Delta$ (in the rest frame of the pulse), where $\Delta=$ $\operatorname{sech}^{-1}\left(2^{-1 / 2}\right) \simeq 0.8814$ is scale factor, are described by $\rho_{\mathrm{b}}\left(\Theta_{\mathrm{b}}=\nu \Delta\right)=\rho_{0} / 2$ [34]. Applying that condition to solution $(27 \mathrm{~b})$ shows that $\rho_{0}$ and $\rho_{\text {sat }}$ can be connected 


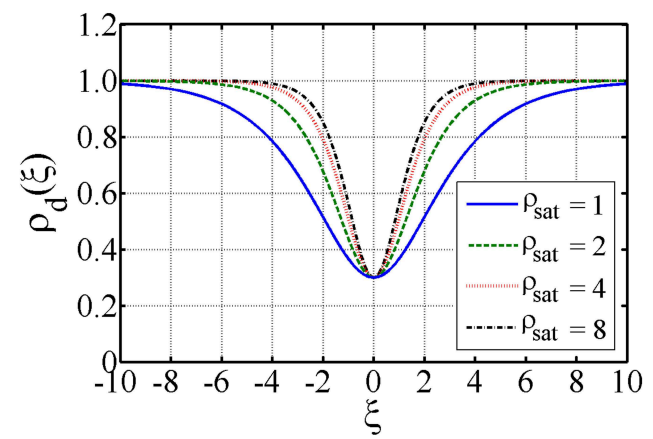

FIG. 2: (color online) Dark soliton intensity distributions obtained by solving Eq. (19a) with $\rho_{0}=1.0$ and $\rho_{1}=0.3$. These profiles are universal since they are insensitive to $\kappa$, $\alpha$, and $V_{0 \mathrm{~b}}$. The contraction (pulse narrowing) appears for normal GVD $(s=-1)$ when considering these curves as functions of $\tau$ rather than of $\xi[12]$.

by the implicit equation [29]

$$
\begin{aligned}
\tan ^{-1}\left[\left(\frac{\rho_{0} / \rho_{\text {sat }}}{2+\rho_{0} / \rho_{\text {sat }}}\right)^{1 / 2}\right] \\
+\frac{1}{2}\left(\frac{\rho_{\text {sat }}}{\rho_{0}}\right)^{1 / 2} \ln \left[\frac{\left(2+\rho_{0} / \rho_{\text {sat }}\right)^{1 / 2}+1}{\left(2+\rho_{0} / \rho_{\text {sat }}\right)^{1 / 2}-1}\right] \\
=\left(\frac{\rho_{\text {sat }}}{1+\rho_{0} / \rho_{\text {sat }}}\right)^{1 / 2} \nu \Delta .
\end{aligned}
$$

For weak saturation effects, the lower branch tends towards $\rho_{0} \simeq 1 / \nu^{2}$ while for strong saturation [characterized by $\left.\rho_{0} / \rho_{\text {sat }} \gg \mathcal{O}(1)\right]$, the upper branch diverges according to $\rho_{0} \simeq \rho_{\text {sat }}\left[\rho_{\text {sat }}(4 \nu \Delta / \pi)^{2}-1\right]$ (see Fig. 3 ).

For dark solitons, the bistability condition changes slightly to read $\rho_{\mathrm{d}}\left(\Theta_{\mathrm{d}}=\nu \Delta\right)=\left(\rho_{0}+\rho_{1}\right) / 2$ [35], in which case application to solution (28b) leads to a second implicit equation [30]

$$
\begin{aligned}
& \left(\frac{\rho_{\mathrm{sat}}}{\rho_{0}}\right)^{1 / 2} \frac{\left(1+\rho_{0} / \rho_{\mathrm{sat}}\right)^{1 / 2}}{A} \\
& \times \tanh ^{-1}\left[\left(\frac{\rho_{\mathrm{sat}}}{\rho_{0}}\right)^{1 / 2}\left(\frac{1+\rho_{0} / \rho_{\mathrm{sat}}}{2-A^{2}+2 \rho_{\mathrm{sat}} / \rho_{0}}\right)^{1 / 2}\right] \\
& -\tanh ^{-1}\left[\frac{A}{\left(2-A^{2}+2 \rho_{\mathrm{sat}} / \rho_{0}\right)^{1 / 2}}\right] \\
& =\frac{\rho_{\mathrm{sat}}^{1 / 2}}{\left(1+\rho_{0} / \rho_{\mathrm{sat}}\right)\left[1+\left(1-A^{2}\right) \rho_{0} / \rho_{\mathrm{sat}}\right]^{1 / 2}} \nu \Delta .
\end{aligned}
$$

Equation (29b) prescribes pairs of gray solitons that have the same FWHM but where the cw backgrounds have different intensities. The lower branch tends towards $\rho_{0} \simeq 1 / \nu^{2} A^{2}$ in the weak saturation regime (see Fig. 4), as expected [30].

\section{ASYMPTOTIC ANALYSES}

\section{A. Soliton hierarchies}

For waves of low intensity, defined by $|u|^{2} / \rho_{\text {sat }} \ll \mathcal{O}(1)$, saturation is relatively weak and the system response is traditionally represented through a truncated power series [18]. To leading order, the dominant nonlinear contribution to Eq. (1) is well approximated by

$$
\frac{1}{2} \frac{2+|u|^{2} / \rho_{\mathrm{sat}}}{\left(1+|u|^{2} / \rho_{\mathrm{sat}}\right)^{2}}|u|^{2} u \simeq\left(1-\frac{3}{2 \rho_{\mathrm{sat}}}|u|^{2}\right)|u|^{2} u,
$$

which corresponds to a cubic-quintic model with a dominant (positive) cubic term and a small (negative) quintic correction. In the notation of Ref. [12], one can identify coefficients $\gamma_{2} \equiv+1$ and $\gamma_{4} \equiv-3 / 2 \rho_{\text {sat }}$ with the standard cubic nonlinearity clearly recovered when the quintic contribution is neglected. Hence, we expect spatiotemporal saturable solitons to transition through their cubic-quintic countparts when intensities are much less than $\rho_{\text {sat }}$ and the cubic limit is approached. It is selfevident that the underlying quadrature equations must also reduce accordingly at each stage.

\section{Bright solitons}

To facilitate the asymptotic analysis of bright solitons, it is helpful to recast exact solution (27b) in the slightly different but more instructive form [37]

$$
\begin{gathered}
2\left(\frac{\rho_{0}}{\rho_{\mathrm{sat}}}\right)^{1 / 2} \tan ^{-1}\left[\left(\frac{\rho_{0}}{\rho_{\mathrm{sat}}}\right)^{1 / 2}\left(\frac{1-\rho_{\mathrm{b}} / \rho_{0}}{1+\rho_{\mathrm{b}} / \rho_{\mathrm{sat}}}\right)^{1 / 2}\right] \\
+\cosh ^{-1}\left[\frac{2 \rho_{0}\left(1-\rho_{0} / \rho_{\mathrm{sat}}\right)^{-1}-\rho_{\mathrm{b}}}{\rho_{\mathrm{b}}\left(1+\rho_{0} / \rho_{\mathrm{sat}}\right)\left(1-\rho_{0} / \rho_{\mathrm{sat}}\right)^{-1}}\right] \\
=2 \sqrt{2 \beta_{\mathrm{b}}} \Theta_{\mathrm{b}}(\tau, \zeta),
\end{gathered}
$$

which more closely resembles the target cubic-quintic bright soliton [12]. For $\rho_{0} / \rho_{\text {sat }} \ll \mathcal{O}(1)$, result (31a) is well approximated by

$$
\begin{aligned}
& 2\left(\frac{\rho_{0}}{\rho_{\mathrm{sat}}}\right)\left(1-\frac{\rho_{\mathrm{b}}}{\rho_{0}}\right)^{1 / 2} \\
& \quad+\cosh ^{-1}\left[\frac{2 \rho_{0}\left(1+\rho_{0} / \rho_{\mathrm{sat}}\right)-\rho_{\mathrm{b}}}{\rho_{\mathrm{b}}\left(1+2 \rho_{0} / \rho_{\mathrm{sat}}\right)}\right] \simeq 2 \sqrt{2 \beta_{\mathrm{b}}} \Theta_{\mathrm{b}}(\tau, \zeta) .
\end{aligned}
$$

In going from Eq. (31a) to (31b), one is obliged to recognize the leading-order change to the $\beta_{\mathrm{b}}$ parameter [c.f. Eq. (7c)], namely $\beta_{\mathrm{b}} \simeq\left(1-\rho_{0} / \rho_{\mathrm{sat}}\right)\left(\rho_{0} / 2\right)$. Hence, the net velocity $W_{\mathrm{b}}$ [defined in Eq. (25b)] has the correct behaviour so that the right-hand side of Eq. (31b) naturally converges. Note that $\rho_{0} / \rho_{\text {sat }}$ does not need to be all that small before the left-hand side of Eq. (31b) can 
be replaced to yield:

$$
\cosh ^{-1}\left[\frac{2 \rho_{0}\left(1-\rho_{0} / \rho_{\mathrm{sat}}\right)-\rho_{\mathrm{b}}}{\rho_{\mathrm{b}}\left(1-2 \rho_{0} / \rho_{\mathrm{sat}}\right)}\right] \simeq 2 \sqrt{2 \beta_{\mathrm{b}}} \Theta_{\mathrm{b}}(\tau, \zeta)
$$

[for instance, approximate solutions (31b) and (31c) are nearly indistinguishable for $\left.\rho_{0} / \rho_{\text {sat }}=1 / 10\right]$. Crucially, it follows that the approximate saturable soliton described by Eq. (31c) corresponds to an exact cubic-quintic soliton with $\gamma_{2}=+1$ and $\gamma_{4} \equiv-3 / 2 \rho_{\text {sat }}[12]$. As $\rho_{0} / \rho_{\text {sat }} \rightarrow 0$, the cubic result also emerges from Eq. (31c) [11],

$$
\cosh ^{-1}\left(\frac{2 \rho_{0}-\rho_{\mathrm{b}}}{\rho_{\mathrm{b}}}\right) \simeq 2 \rho_{0}^{1 / 2} \Theta_{\mathrm{b}}(\tau, \zeta),
$$

as it must. Hence we have proved that bright solitons form a hierarchy wherein saturable solutions must necessarily pass through the corresponding cubic-quintic shape before finally (and rather slowly) converging on the cubic limit as $\rho_{0} / \rho_{\text {sat }} \rightarrow 0$.

\section{Dark solitons}

A similar analysis of dark solitons is more involved, but the same procedure is generally followed for its intensity and phase quadratures. Exact solution (28b) describing the intensity distribution can be reexpressed [37] as

$$
\begin{aligned}
\cosh ^{-1}\left\{\frac{2 \rho_{0} A^{2}\left(1+\rho_{0} / \rho_{\mathrm{sat}}\right)-\left[1+\left(1+A^{2}\right) \rho_{0} / \rho_{\mathrm{sat}}\right]\left(\rho_{0}-\rho_{\mathrm{d}}\right)}{\left[1+\left(1-A^{2}\right) \rho_{0} / \rho_{\mathrm{sat}}\right]\left(\rho_{0}-\rho_{\mathrm{d}}\right)}\right\} \\
-\frac{2 A}{\left(1+\rho_{0} / \rho_{\mathrm{sat}}\right)^{1 / 2}}\left(\frac{\rho_{0}}{\rho_{\mathrm{sat}}}\right)^{1 / 2} \tanh ^{-1}\left\{\frac{1}{\rho_{\mathrm{sat}}^{1 / 2}}\left[\frac{\rho_{\mathrm{d}}-\left(1-A^{2}\right) \rho_{0}}{1+\rho_{\mathrm{d}} / \rho_{\mathrm{sat}}}\right]^{1 / 2}\right\}=2 \sqrt{2 \beta_{\mathrm{d}}} \Theta_{\mathrm{d}}(\tau, \zeta),
\end{aligned}
$$

and where we have introduced the parameter

$$
\beta_{\mathrm{d}} \equiv \frac{\rho_{0} A^{2}}{2}\left(1+\frac{\rho_{0}}{\rho_{\mathrm{sat}}}\right)^{-3}\left[1+\left(1-A^{2}\right) \frac{\rho_{0}}{\rho_{\mathrm{sat}}}\right]^{-1}
$$

for a more transparent recovery of known results for the cubic-quintic system [12].

In the limit $\rho_{0} / \rho_{\text {sat }} \ll \mathcal{O}(1)$, the expression for $\beta_{\mathrm{d}}$ re- duces to its cubic-quintic counterpart with $\gamma_{2}=+1$ and $\gamma_{4}=-3 / 2 \rho_{\text {sat }}$, namely $\beta_{\mathrm{d}}=\left(\rho_{0} A^{2} / 2\right)\left[1-\left(4-A^{2}\right) \rho_{0} / \rho_{\text {sat }}\right]$ [12]. Since the intrinsic velocity $V_{0 d}$ derived in Ref. [12] is also recovered from Eq. (21a) in the same way, it follows that right-hand side of Eq. (32a) necessarily converges as desired. As in the case of bright solitons, the ratio $\rho_{0} / \rho_{\text {sat }}$ does not need to be especially small before the exact left-hand side of Eq. (32a) can be replaced with an excellent approximation thus:

$$
\cosh ^{-1}\left\{\frac{2 \rho_{0} A^{2}\left[1-\left(\rho_{0} / \rho_{\mathrm{sat}}\right)\left(4-A^{2}\right)\right]-\left(1-4 \rho_{0} / \rho_{\mathrm{sat}}\right)\left(\rho_{0}-\rho_{\mathrm{d}}\right)}{\left[1-2\left(\rho_{0} / \rho_{\mathrm{sat}}\right)\left(2-A^{2}\right)\right]\left(\rho_{0}-\rho_{\mathrm{d}}\right)}\right\} \simeq 2 \sqrt{2 \beta_{\mathrm{d}}} \Theta_{\mathrm{d}}(\tau, \zeta)
$$

One subsequently recovers the cubic-quintic intensity profile detailed in Ref. [12]. As $\rho_{0} / \rho_{\text {sat }} \rightarrow 0$, the wellknown profile for the cubic system [7] appears as the limit of Eq. (33a):

$$
\cosh ^{-1}\left[\frac{2 \rho_{0} A^{2}-\left(\rho_{0}-\rho_{\mathrm{d}}\right)}{\rho_{0}-\rho_{\mathrm{d}}}\right] \simeq 2 \rho_{0}^{1 / 2} A \Theta_{\mathrm{d}}(\tau, \zeta) .
$$

The phase distribution of the cubic-quintic dark soliton can be recovered from the saturable solution in a similar way (we do not present the details here). Hence, dark solitons must also form a self-consistent saturable - cubicquintic - cubic hierarchy.

\section{B. Slowly-varying envelopes}

\section{Envelope equation}

By neglecting the first term in Eq. (1), we can recover the governing equation of conventional pulse theory in the laboratory frame:

$$
i\left(\frac{\partial u}{\partial \zeta}+\alpha \frac{\partial u}{\partial \tau}\right)+\frac{s}{2} \frac{\partial^{2} u}{\partial \tau^{2}}+\frac{1}{2} \frac{2+|u|^{2} / \rho_{\mathrm{sat}}}{\left(1+|u|^{2} / \rho_{\mathrm{sat}}\right)^{2}}|u|^{2} u \simeq 0 .
$$

By Galilean boosting to the local time-frame, defined by coordinates $\tau_{\text {loc }} \equiv \tau-\alpha \zeta$ and $\zeta_{\text {loc }}=\zeta$, Eq. (34a) is transformed into the more familiar form considered by 


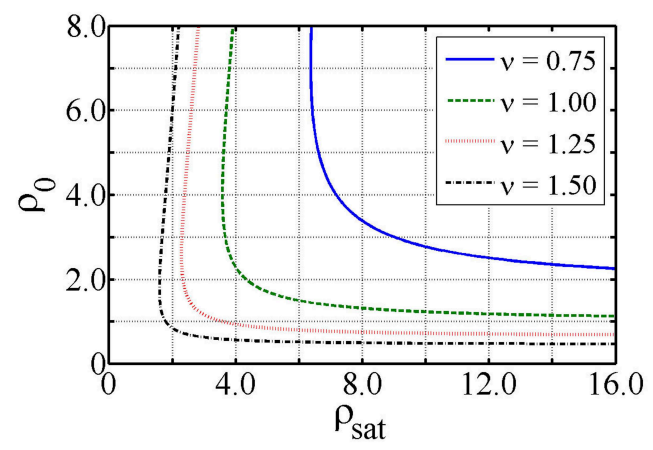

FIG. 3: (color online) Non-degenerate bistablity curves for saturable bright solitons as predicted by Eq. (29a). Lower branches with $1 / \rho_{\mathrm{sat}} \rightarrow 0$ correspond to regimes where saturation is weak, in which case the peak intensity converges toward $\rho_{0} \simeq 1 / \nu^{2}$.

Krolikowski and Luther-Davies [29, 30],

$$
i \frac{\partial u}{\partial \zeta_{\mathrm{loc}}}+\frac{s}{2} \frac{\partial^{2} u}{\partial \tau_{\mathrm{loc}}^{2}}+\frac{1}{2} \frac{2+|u|^{2} / \rho_{\mathrm{sat}}}{\left(1+|u|^{2} / \rho_{\mathrm{sat}}\right)^{2}}|u|^{2} u \simeq 0
$$

Crucially, one must be able to recover the soliton solutions of these two related models in the limit that all contributions from $\kappa \partial^{2} u / \partial \zeta^{2}$ are sufficiently small simultaneously. In practice, one is obliged to asymptote the spatiotemporal predictions for velocities and propagation constants in conjunction with a Galilean transformation to the $\left(\tau_{\text {loc }}, \zeta_{\text {loc }}\right)$ frame.

\section{Intrinsic, transverse, and net velocities}

We first consider algebraic results for the various velocity contributions (10b), (21b), and (25a) under the assumption of slowly-varying envelopes. The label 'SVEA' is used to denote these components in the laboratory frame, while 'loc' refers to their local-time frame representations.

In the limit $\kappa \beta_{\mathrm{b}} \ll \mathcal{O}(1)$, bright solitons have an intrinsic velocity $V_{0 \mathrm{~b}} \simeq \alpha \equiv V_{0 \mathrm{~b} \text { SVEA }}$. For dark solitons with $\kappa \beta_{\text {cw }} \ll \mathcal{O}(1)$ and $\kappa \alpha^{2} \ll \mathcal{O}(1)$, it follows that $V_{0 \mathrm{~d}} \simeq V_{0 \mathrm{~d} \text { loc }}+\alpha \equiv V_{0 \mathrm{~d} \text { SVEA }}$. When considering the parameters $V_{\mathrm{b}, \mathrm{d}}$, the additional inequality $\kappa|\Omega(\alpha+\Omega / 2)| \ll$ $\mathcal{O}(1)$ leads to the simple result $V_{\mathrm{b}, \mathrm{d}} \simeq \Omega \equiv V_{\text {SVEA }}$. This key finding demonstrates that transverse velocities and frequency shifts are interchangeable in conventional pulse theory. Finally, the net velocities $W_{\mathrm{b}, \mathrm{d}}$ are well-approximated by $W_{\mathrm{b}, \mathrm{d}} \simeq V_{0 \mathrm{~b}, 0 \mathrm{~d} \text { SVEA }}+V_{\mathrm{SVEA}} \equiv$ $W_{\mathrm{b}, \mathrm{d} \text { SVEA }}$.

In the local time-frame, parameters $V_{0 \mathrm{~b}, 0 \mathrm{~d} \text { SVEA }}$ and $V_{\text {SVEA }}$ combine in a way that maps directly onto the velocity combination rule of Galilean relativity. For bright solitons, the intrinsic velocity $V_{0 \mathrm{bSVEA}}$ is transformed away so that in $\left(\tau_{\text {loc }}, \zeta_{\text {loc }}\right)$ coordinates one has $V_{0 b \text { loc }}=0$. It then follows that $W_{\mathrm{bloc}}=V_{\mathrm{SVEA}}=\Omega$, and hence solutions with $\Omega=0$ describe pulses that are strictly stationary (that is, they travel with their peak always centered
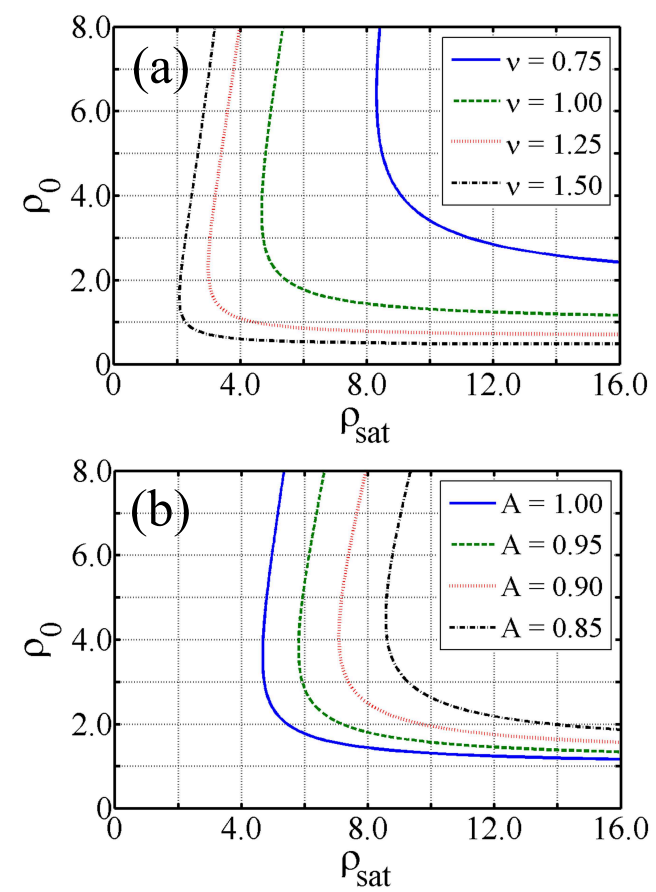

FIG. 4: (color online) Non-degenerate bistablity curves for saturable dark solitons [(a) black $(A=1)$ and $(\mathrm{b})$ gray (with $\nu=1$ ) solutions] as predicted by Eq. (29b). Lower branches with $1 / \rho_{\text {sat }} \rightarrow 0$ correspond to weak saturation, in which case the cw intensity tends to $\rho_{0} \simeq 1 / \nu^{2} A^{2}$.

on $\left.\tau_{\text {loc }}=0\right)$. The situation is slightly more complicated for dark solitons. The factor $\alpha$ in $V_{0 \mathrm{~d} \text { SVEA }}$ disappears so that the intrinsic velocity of the dark soliton is simply $V_{0 \mathrm{~d} \text { loc }}$ and hence $W_{\mathrm{d} \text { loc }}=V_{0 \mathrm{~d} \text { loc }}+\Omega$. Black solutions (having $F=0=V_{0 \text { d loc }}$ ) with $\Omega=0$ thus have zero local net velocity and are also strictly stationary.

\section{Asymptotic solutions}

Since all the coordinate transformations we have considered here are geometrical operations, the profile of any solution must be independent of the frame of reference in which it is observed. For example, a sech-shaped pulse in the laboratory frame must also be sech-shaped in the local-time frame (though in spatiotemporal regimes one necessarily encounters a contraction or dilation factor) $[6,7]$.

From the results of the previous subsection, it follows that under the SVEA, one must have $\Theta_{\mathrm{b}, \mathrm{d}}(\tau, \zeta) \simeq$ $\tau \mp W_{\mathrm{b}, \mathrm{d}} \zeta \equiv \Theta_{\mathrm{b}, \mathrm{d} \operatorname{SVEA}}(\tau, \zeta)$. The intensity and phase quadratures of localized components of the solutions [c.f., Eqs. (27b), (28a) and (28b)] remain formally unchanged when assuming slowly-varying envelopes (one simply replaces $\Theta_{b, d}$ with $\Theta_{b, d}$ SVEA $)$. However, the linear part of the phase distribution alters its structure due to the coordinate change. When considering forward-propagating spatiotemporal solitons, transforming to the local-time 
frame shows that

$$
u_{\mathrm{b}}\left(\tau_{\mathrm{loc}}, \zeta_{\mathrm{loc}}\right) \propto \exp \left[i \Omega \tau_{\mathrm{loc}}+i\left(\beta_{\mathrm{b}}-\frac{\Omega^{2}}{2}\right) \zeta_{\mathrm{loc}}\right],
$$

and

$$
u_{\mathrm{d}}\left(\tau_{\text {loc }}, \zeta_{\text {loc }}\right) \propto \exp \left[-i \Omega \tau_{\text {loc }}+i\left(\beta_{\mathrm{cw}}+\frac{\Omega^{2}}{2}\right) \zeta_{\text {loc }}\right] .
$$

These solutions satisfy Eq. (34b) exactly for $s=+1$ and $s=-1$, respectively. Hence, one can now fully appreciate that the known (conventional) solitons derived by Krolikowski and Luther-Davies [29, 30] are important subsets of the more general spatiotemporal solutions. Applying the same multiple-limit procedure to the backward spatiotemporal solitons yields largely similar results except that a rapidly-varying phase term, $\exp [-i 2(\zeta / 2 \kappa)]$, survives in the linear phase distributions. The non-vanishing nature of this contribution demonstrates that conventional pulse theory (based on a nonlinear-Schrödinger formalism) has no analogue of backward waves.

\section{SOLITON STABILITY}

In going from cubic [7] to cubic-quintic [12] to saturable systems, the linear part of the wave operator always has the same form. In that sense, one does not expect to encounter substantial changes in soliton stability characteristics since the fundamental elliptic / hyperbolic structure of the governing equation remains unchanged. Here, the same physical arguments with regards frame-of-reference symmetries are deployed, and standard tools [viz., the Vakhitov-Kolokolov (VK) and renormalized-momentum integral criteria] that have previously proved so invaluable $[7,12]$ are applied to make predictions about the robustness of localized solutions to Eq. (1).

\section{A. Vakhitov-Kolokolov criterion}

A bright-type solution of Eq. (34b) is predicted to be stable against small disturbances if the integrated pulse intensity $P$, defined by

$$
P \equiv \int_{-\infty}^{+\infty} d \tau_{\mathrm{loc}}\left|u_{\mathrm{b}}\right|^{2}
$$

has a positive gradient such that

$$
\frac{d}{d \beta_{\mathrm{b}}} P\left(\beta_{\mathrm{b}}\right)>0
$$

where $\beta_{\mathrm{b}}$ is the propagation constant [c.f. Eq. (7c)]. Since the wave intensity profile is known only implicitly, the explicit computation of $P\left(\beta_{\mathrm{b}}\right)$ can be potentially awkward.

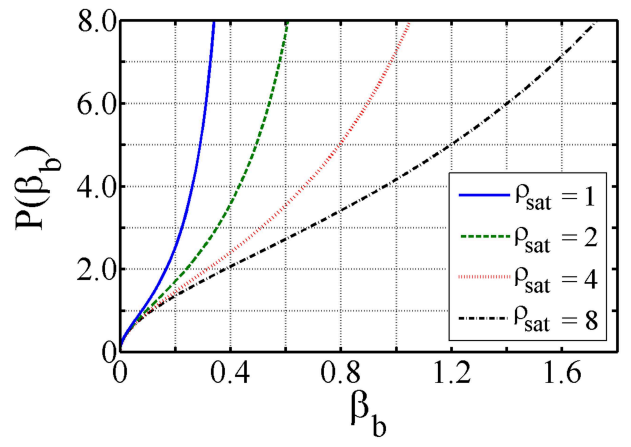

FIG. 5: (color online) Integrated intensity curves $P\left(\beta_{\mathrm{b}}\right)$ obtained from Eq. (36c) for increasing saturation parameter.

The gradient $d P / d \beta_{\mathrm{b}}$ is always positive so that the VK criterion [c.f. Eqs. (36a) and (36b)] is satisfied.

However, the calculation can be facilitated indirectly by transforming the integral [36] so that

$$
P\left(\beta_{\mathrm{b}}\right)=\frac{1}{\sqrt{2}} \int_{0}^{\rho_{0}\left(\beta_{\mathrm{b}}\right)} d R_{0}\left[\beta_{\mathrm{b}}-\frac{1}{1+R_{0} / \rho_{\mathrm{sat}}}\left(\frac{R_{0}}{2}\right)\right]^{-1 / 2},
$$

where $\rho_{0}\left(\beta_{\mathrm{b}}\right)=2 \beta_{\mathrm{b}} /\left(1-2 \beta_{\mathrm{b}} / \rho_{\text {sat }}\right)$. Since the peak intensity lies in the range $0 \leq \rho_{0}<\infty$, it must be that $0 \leq \beta_{\mathrm{b}}<\rho_{\text {sat }} / 2$ (note that the corresponding cubicquintic soliton also has a maximum allowed value for $\beta_{\mathrm{b}}$ [12]). Plotting the $P\left(\beta_{\mathrm{b}}\right)$ curves reveals that inequality (36b) tends to be satisfied and hence saturable solitons are anticipated to be stable entities (see Fig. 5).

\section{B. Perturbed bright solitons}

To test analytical predictions of bright soliton stability, a similar prescription is followed to that in Ref. [12]. Initial data is selected for Eq. (1) using solution (27a) but where the dilation factor $\left(1+2 \kappa W_{\mathrm{b}}^{2}\right)^{1 / 2}$ characterizing the broadening of $\rho_{\mathrm{b}}(\tau, 0)$ in the anomalous-GVD regime is omitted from $\Theta_{\mathrm{b}}(\tau, 0)$. The strength of the local shape perturbation thus increases with $\Omega=4,8,12$ and 16 .

Typical examples of pulse self-reshaping due to internal dynamics are shown in Fig. 6. A saturation intensity of $\rho_{\text {sat }}=4.0$ has, according to Eq. (29a), bistable lower- and upper-branch solitons with peak intensities $\rho_{0} \simeq 2.298$ and $\rho_{0} \simeq 8.763$, respectively. The lower-branch solution exhibits monotonically-decaying oscillations as the waveform evolves towards a stationary state of Eq. (1), with a small amount of energy shed as low-amplitude dispersive waves (radiation). The upper-branch solution behaves somewhat differently, with persistent (and much more rapid) oscillations surviving in the long-term evolution. These oscillations are bounded within an envelope that is weakly modulated in $\zeta$-behaviour that is consistent with the excitation of an internal mode [38] — with simulations giving no indication of convergence towards a 
stationary state (even over propagation distances much longer than those shown).

The self-reshaping characteristics of the lower-branch soliton are reminiscent of those recently uncovered in cubic-quintic systems [12]. By interpreting perturbationinduced radiative losses as a mechanism for local energy dissipation (while noting that the system is still globally conservative), one might regard the asymptotic stationary states emerging in Fig. 6(a) as fixed-point attractors surrounded by wide basins of attraction. Similarly, the surviving oscillatory solutions in Fig. 6(b) are qualitatively similar to the limit-cycle attractors reported elsewhere in spatial soliton contexts [7].

\section{Renormalized-momentum criterion}

The stability of dark solitons of generalized NLS-type models such as Eq. (34b) is typically discussed in terms of a renormalized momentum $M_{\text {ren }}$,

$$
M_{\text {ren }} \equiv \frac{i}{2} \int_{-\infty}^{+\infty} d \tau_{\text {loc }}\left(u_{\mathrm{d}} \frac{\partial u_{\mathrm{d}}^{*}}{\partial \tau_{\mathrm{loc}}}-u_{\mathrm{d}}^{*} \frac{\partial u_{\mathrm{d}}}{\partial \tau_{\mathrm{loc}}}\right)\left(1-\frac{\rho_{0}}{\left|u_{\mathrm{d}}\right|^{2}}\right)
$$

The integral expression for $M_{\text {ren }}$ can be recast in a more convenient form by way of Eq. (14a) and by noting that
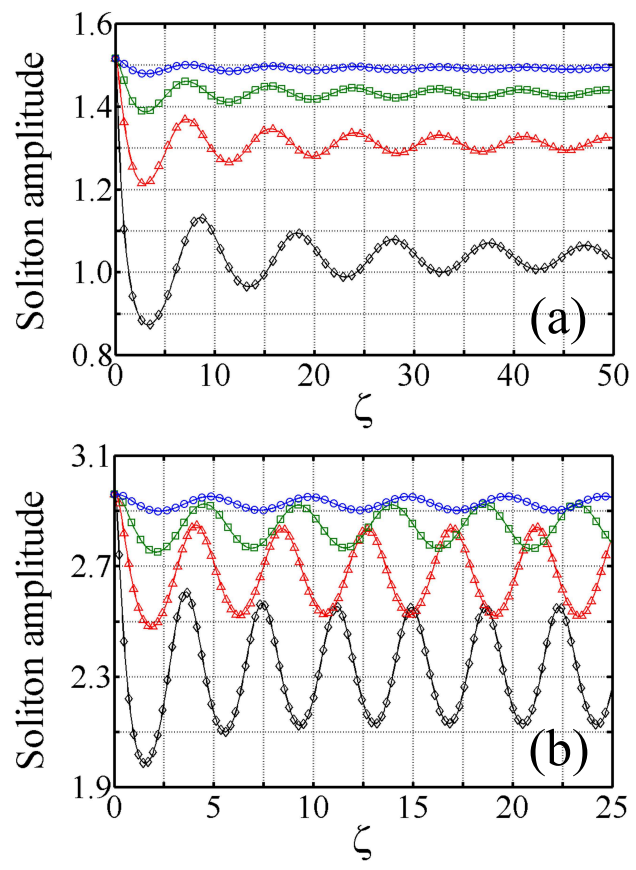

FIG. 6: (color online) Evolution of the bistable bright soliton peak amplitude when the initial waveform resides on the (a) lower branch $\left(\rho_{0}=2.298\right)$ and (b) upper branch $\left(\rho_{0}=8.763\right)-$ c.f. Fig. 3 with $\nu=1.0$. System parameters: $\rho_{\text {sat }}=4.0, s=+1, \alpha=1.0, \kappa=1.0 \times 10^{-3}$. Blue circle:

$\Omega=4$. Green square: $\Omega=8$. Red triangle: $\Omega=12$. Black diamond: $\Omega=16$. $\xi$ and $\tau_{\text {loc }}$ are interchangeable in conventional analyses:

$$
M_{\text {ren }}=-V_{0 \mathrm{~d} \text { loc }} \int_{-\infty}^{+\infty} d \tau_{\text {loc }} \frac{\left(\rho_{\mathrm{d}}-\rho_{0}\right)^{2}}{\rho_{\mathrm{d}}}
$$

Solitons are predicted to be stable when the inequality

$$
\frac{d}{d V_{0 \mathrm{~d} \text { loc }}} s M_{\text {ren }}\left(V_{\text {Od loc }}\right)>0
$$

is satisfied. Note that Eq. (37c), proved by Barashenkov [39] to be an acceptable stability criterion and further developed by Pelinovsky et al. [40], captures nonlinear dynamical phenomena that the MI (linear) calculation of Sec. IV A (and Appendix C) clearly cannot.

By considering the behaviour of the integral in Eq. (37b), Kivshar and Afanasjev have shown that black solitons of Eq. (34b) possess a drift instability that appears when $\rho_{\text {sat }} / \rho_{0}$ is less than a critical value of $\approx 0.45$ (see Fig. 3 in Ref. [41]). The instability manifests itself as the gradual transformation of an initially black $(F=0)$ solution into a gray $(|F|>0)$ waveform, accompanied by the strong emission of radiation. In the following simulations with Eq. (1), we restrict our attention to parameter regimes above criticality. A set of typical curves for the renormalized momentum, as defined in Eq. (37b), is shown in Fig. 7. The gradients are generally positive and $M_{\text {ren }}$ is undefined at $V_{0 \mathrm{~d} \text { loc }}=0[41]$.

\section{Perturbed dark solitons}

The perturbed dark soliton initial-value problem is defined by using solution (28a) but where the contraction factor $\left(1-2 \kappa W_{\mathrm{d}}^{2}\right)^{1 / 2}$ in the normal-GVD regime is omitted from $\Theta_{\mathrm{d}}(\tau, 0)$. Attention is first paid to black solitons with frequency shifts of $\Omega=4,8,12$, and 16 . The temporal width of the input pulse, defined as $w_{0} \equiv\left(2 \beta_{\mathrm{d}}\right)^{-1 / 2}$

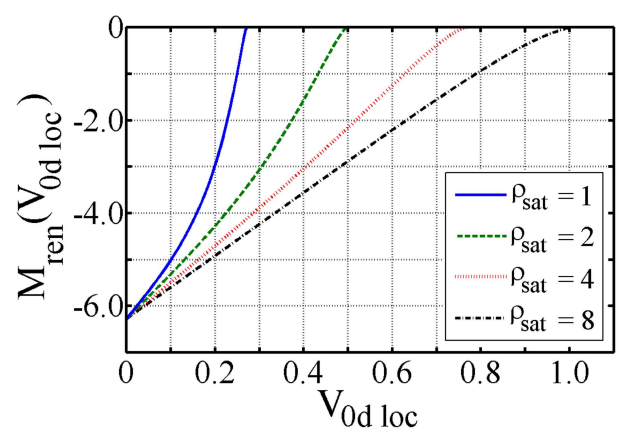

FIG. 7: (color online) Renormalized dark soliton momentum when $\rho_{0}=2.0$, computed with Eq. (37b) for the contrast range $0<F \leq 1$. The gradient $d M_{\text {ren }} / d V_{0 \mathrm{~d} \text { loc }}$ is always positive for $V_{0 \mathrm{~d} \text { loc }}>0$, so that stability criterion (37c) is always satisfied. Here, $M_{\text {ren }}$ approaches a numerical value of $-2 \pi$ as $V_{0 d \text { loc }}$ tends toward zero. 

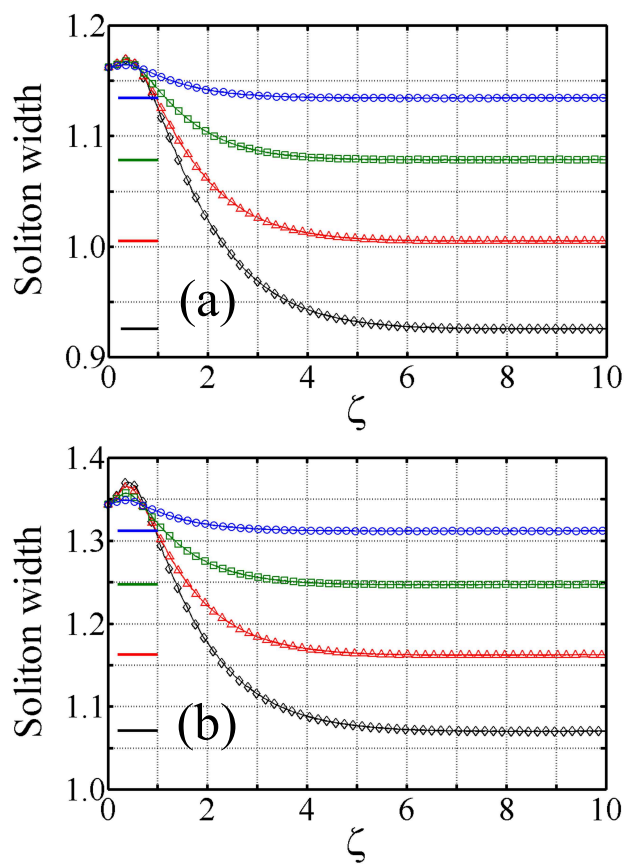

FIG. 8: (color online) Evolution of the bistable black soliton full width when the initial waveform resides on the

(a) lower branch $\left(\rho_{0}=2.383\right)$ and (b) upper branch $\left(\rho_{0}=6.167\right)$ - c.f. Fig. 4 with $\nu=1.0$ (horizontal bars indicate theoretical predictions). System parameters: $\rho_{\text {sat }}=5.0, s=-1, \alpha=1.0, \kappa=1.0 \times 10^{-3}$. Blue circle: $\Omega=4$. Green square: $\Omega=8$. Red triangle: $\Omega=12$. Black diamond: $\Omega=16$.

[c.f. Eq. (32b)], is broader than that required by the exact solution. We thus expect the localized pulse to become narrower as it travels through space, evolving smoothly towards its limiting value of $w_{\infty}=w_{0}\left(1-2 \kappa W_{\mathrm{d}}^{2}\right)^{1 / 2}$. Typical self-reshaping characteristics are shown in Fig. 8 for $\rho_{\text {sat }}=5.0$ and $\nu=1.0$ [in which case Eq. (29b) determines the lower- and upper-branch $\mathrm{cw}$ intensities to be $\rho_{0} \simeq 2.383$ and $\rho_{0}=6.167$, respectively]. The evolution into a stationary state occurs relatively rapidly, within a distance of $\zeta \simeq 10$.

Finally, illustrative results are presented for perturbed gray solitons (curves for the reshaping pulse widths are similar to those in Fig. 8 but they occur on much longer scalelengths in $\zeta$ ). Such solutions tend not to preserve their grayness and are described by $F \rightarrow F(\zeta)$, where $F(\zeta)$ must be obtained numerically. Gray solitons thus often exhibit a small drift-type instability that is reminiscent of that in cubic-quintic systems [12], where the minimum intensity of the pulse dip (which is itself varying slowly in $\zeta$ ) tends to travel along the (approximately linear) characteristic $\tau-W_{\mathrm{d}}(\zeta) \zeta=$ const. and where $V_{0 \mathrm{~d}}(\zeta)$ is computed from Eq. (21a) after adiabatic fitting of the numerical dataset to solution (28b). Typical variations in relaxing grayness for perturbed solitons with a fixed saturation intensity $\rho_{\text {sat }}=5.0$ are shown in Fig. 9. Simulations have also shown that for a fixed perturbation

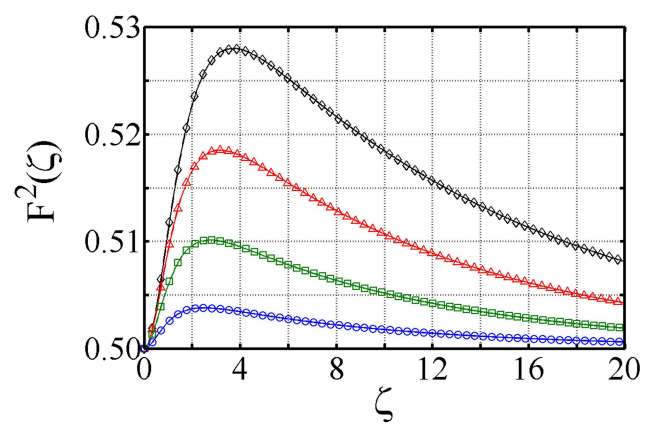

FIG. 9: (color online) Evolution of the gray soliton contrast parameter when the initial waveform has $\rho_{0}=2.0$ and $F^{2}(0)=0.5$. System parameters: $\rho_{\text {sat }}=5.0, s=-1$, $\alpha=1.0, \kappa=1.0 \times 10^{-3}$. Blue circle: $\Omega=4$. Green square: $\Omega=8$. Red triangle: $\Omega=12$. Black diamond: $\Omega=16$.

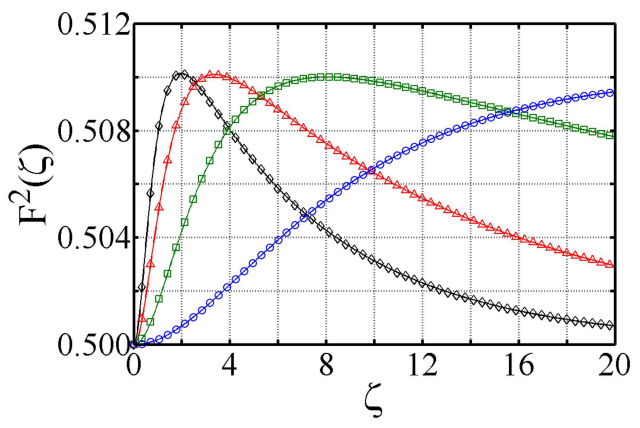

FIG. 10: (color online) Evolution of the gray soliton contrast parameter when the initial waveform has $\rho_{0}=2.0$ and $F^{2}(0)=0.5$ and the initial perturbation has $\Omega=8.0$. System parameters: $s=-1, \alpha=1.0, \kappa=1.0 \times 10^{-3}$. Blue circle: $\rho_{\text {sat }}=1.0$. Green square: $\rho_{\text {sat }}=2.0$. Red triangle: $\rho_{\text {sat }}=4.0$. Black diamond: $\rho_{\text {sat }}=8.0$.

strength (e.g., for a moderate value $\Omega=8.0$ ), the relaxation of $F(\zeta)$ tends to occur over distances that decrease with increasing $\rho_{\text {sat }}$ (see Fig. 10).

\section{CONCLUSIONS}

Our analyses of spatiotemporal systems have now considered exact bright and dark envelope solitons in some detail for the three classic nonlinearities where one might expect to find analytical solutions - cubic [11], cubicquintic [12], and saturable models. These wavepackets tend to exhibit a raft of corrections to their known conventional counterparts that arise solely from the spacetime-symmetric nature of the governing equation; they include relativistic- or pseudorelativistic-type contraction/dilation factors in the pulse width, along with generic (intensity- and frequency-dependent) modifications to propagation constants and group velocities. In each case, standard mathematical tools have been used to investigate spatiotemporal wave stability, with supporting simulations testing and verifying theoretical pre- 
dictions by way of perturbed initial-value problems. The new solitons have demonstrated remarkable robustness throughout, and they may be interpreted as attractors in the system's dynamics.

Having derived soliton solutions (fundamental stationary states to facilitate subsequent investigations), there is now a wide spectrum of higher-order effects to explore within the spatiotemporal context. Quite general wavebased phenomena to accommodate include third- [42] and fourth-order [43] linear dispersion while, in the photonics domain specifically, self-steepening and stimulated Raman scattering [44, 45] are often practical concerns in optical-fiber systems. The arena of spatiotemporal soliton collisions is also one that merits attention, given the fundamental importance of nonlinear wave interactions in general $[46,47]$ and for optical applications in particular [48].

Finally, it is desirable to extend our $(1+1) \mathrm{D}$ spatiotemporal modelling into higher-dimensional $(2+1) \mathrm{D}$ and $(2+2) \mathrm{D}$ regimes, where excitations may be fully localized in four-dimensional space-time. Such an exercise opens the door to formulating symmetrized (i.e., more complete relativistic- and pseudorelativistic-type) descriptions of exotic phenomena such as optical bullets [8] and $\mathrm{X}$ waves [49] that include the interplay between nonlinearity, GVD, and Helmholtz (as opposed to paraxial) diffraction.

\section{Appendix A: Example in waveguide optics}

We consider a scalar electric field of the form $E(t, z)=$ $A(t, z) \exp \left[i\left(k_{0} z-\omega_{0} t\right)\right]+$ c.c., where $A(t, z)$ is the envelope and "c.c." denotes the complex conjugate of the preceding quantity. The travelling wave has a center frequency $\omega_{0}$ and propagation constant $k_{0}=n_{0} \omega_{0} / c$, where $n_{0}$ is the linear refractive index of the host medium at $\omega_{0}$ and $c$ is the speed of light in vacuo. Taking the wave intensity to be $|A(t, z)|^{2}$, the generic model chosen for a saturable nonlinear refractive index $n_{\mathrm{NL}}$ is [28]

$$
n_{\mathrm{NL}}\left(|A|^{2}\right)=\frac{n_{2} I_{\mathrm{sat}}}{2}\left[1-\frac{1}{\left(1+|A|^{2} / I_{\mathrm{sat}}\right)^{2}}\right],
$$

where $I_{\text {sat }}$ is the saturation intensity parametrizing the response. At low intensities, where $|A|^{2} / I_{\text {sat }} \ll \mathcal{O}(1)$, saturation is small and Eq. (A1) is well approximated by $n_{\mathrm{NL}}\left(|A|^{2}\right) \simeq n_{2}|A|^{2}+n_{4}|A|^{2}$, where $n_{4} \equiv-(3 / 2) n_{2} / I_{\text {sat }}$ (the system essentially has a cubic-quintic response) [18]. Hence, the standard Kerr effect, $n_{\mathrm{NL}}\left(|A|^{2}\right)=n_{2}|A|^{2}$ determines the dominant behaviour when $\left|n_{4}\right||A|^{2}$ is negligible compared with $n_{2}$.

Deployment of the Fourier decomposition techniques to capture the linear-dispersive properties of the system
[16] then leads to the following equation for $A$ :

$$
\begin{aligned}
\frac{1}{2 k_{0}} \frac{\partial^{2} A}{\partial z^{2}} & +i\left(\frac{\partial A}{\partial z}+k_{1} \frac{\partial A}{\partial t}\right)-\frac{k_{2}}{2} \frac{\partial^{2} A}{\partial t^{2}} \\
& +\frac{\omega_{0}}{c}\left(\frac{n_{2}}{2}\right) \frac{2+|A|^{2} / I_{\text {sat }}}{\left(1+|A|^{2} / I_{\text {sat }}\right)^{2}}|A|^{2} A=0 .
\end{aligned}
$$

The two parameters $k_{1} \equiv(\partial k / \partial \omega)_{\omega_{0}}=1 / v_{g}$ and $k_{2} \equiv$ $\left(\partial^{2} k / \partial \omega^{2}\right)_{\omega_{0}}$ are related to the group-velocity and temporal dispersion (GVD), respectively, where $k$ is formally obtained from an associated eigenvalue problem (i.e., solving Maxwell's equations for the transverse distribution of the guided field [50]).

One can now introduce a scaling for the laboratory space $z$ and time $t$ coordinates according to $\zeta=z / L$ and $\tau=t / t_{p}$, and also for the envelope $A(t, z)=A_{0} u(t, z)$. By connecting the spatial and temporal units through $L \equiv t_{p}^{2} /\left|k_{2}\right|$ (essentially scaling to a linearly-dispersing reference Gaussian pulse [50]) and measuring amplitudes in units defined by $\left(\omega_{0} / c\right) n_{2} A_{0}^{2} L \equiv 1$, we can arrive at Eq. (1) where the parameters are $\kappa=1 / 2 k_{0} L, \alpha=$ $k_{1} L / t_{p}, s=-\operatorname{sgn}\left(k_{2}\right)$, and $\rho_{\text {sat }}=I_{\text {sat }} / A_{0}^{2}$.

\section{Appendix B: Two-level atom saturable refractive index model}

\section{Bright solitons}

Perhaps the simplest saturation model stems from the two-level-atom approximation [14]. Here, it is captured within the envelope equation

$$
\kappa \frac{\partial^{2} u}{\partial \zeta^{2}}+i\left(\frac{\partial u}{\partial \zeta}+\alpha \frac{\partial u}{\partial \tau}\right)+\frac{s}{2} \frac{\partial^{2} u}{\partial \tau^{2}}+\frac{1}{1+|u|^{2} / \rho_{\mathrm{sat}}}|u|^{2} u=0,
$$

which is a direct spatiotemporal generalization of the classic model considered by Gatz and Herrmann [25]. For weak saturation, where $|u|^{2} / \rho_{\text {sat }} \ll \mathcal{O}(1)$, the nonlinearity functional tends to the constant $\rho_{\text {sat }}[$ c.f. a limiting value of $\rho_{\text {sat }} / 2$ for Eq. (1)]. Moreover, a competing cubic-quintic response emerges in which $\gamma_{2}=+1$ captures the dominant contribution and $\gamma_{4} \equiv-1 / \rho_{\text {sat }}$ (c.f. $\gamma_{4} \equiv-3 / 2 \rho_{\text {sat }}$ in Sec. VI A) parametrizes the leadingorder correction to the Kerr effect [12, 18].

Following the method detailed in Sec. II, the intensity quadrature is described by

$$
\frac{d}{d \rho_{\mathrm{b}}}\left[\frac{1}{\rho_{\mathrm{b}}}\left(\frac{d \rho_{\mathrm{b}}}{d \xi}\right)^{2}\right]=8\left(\beta_{\mathrm{b}}-\frac{\rho_{\mathrm{b}}}{1+\rho_{\mathrm{b}} / \rho_{\mathrm{sat}}}\right)
$$

so that, after a first integration, we find

$$
\begin{aligned}
\left(\frac{d \rho_{\mathrm{b}}}{d \xi}\right)^{2}= & 8 \beta_{\mathrm{b}} \rho_{\mathrm{b}}^{2}-8 \rho_{\mathrm{sat}} \rho_{\mathrm{b}}^{2}\left[1-\frac{\rho_{\mathrm{sat}}}{\rho_{\mathrm{b}}} \ln \left(1+\frac{\rho_{\mathrm{b}}}{\rho_{\mathrm{sat}}}\right)\right] \\
& +c_{2 \mathrm{~b}} \rho_{\mathrm{b}} .
\end{aligned}
$$


Applying the bright-soliton boundary conditions at $\xi \rightarrow$ $\pm \infty$ shows that $c_{2 \mathrm{~b}}=0$ (recall that the corresponding constant was non-vanishing, assuming a value of $4 \rho_{\text {sat }}^{2}$, in Sec. III A). Considering the boundary conditions at $\xi=0$ also leads to

$$
\beta_{\mathrm{b}}=\rho_{\mathrm{sat}}\left[1-\frac{\rho_{\mathrm{sat}}}{\rho_{0}} \ln \left(1+\frac{\rho_{0}}{\rho_{\mathrm{sat}}}\right)\right] .
$$

Eliminating $\beta_{\mathrm{b}}$ from Eq. (B3) using Eq. (B2b) leads to

$$
\begin{aligned}
\left(\frac{d \rho_{\mathrm{b}}}{d \xi}\right)^{2}= & 8 \rho_{\mathrm{sat}}^{2} \rho_{\mathrm{b}}^{2} \\
& \times\left[\frac{1}{\rho_{\mathrm{b}}} \ln \left(1+\frac{\rho_{\mathrm{b}}}{\rho_{\mathrm{sat}}}\right)-\frac{1}{\rho_{0}} \ln \left(1+\frac{\rho_{0}}{\rho_{\mathrm{sat}}}\right)\right]
\end{aligned}
$$

which cannot now be integrated exactly. To compute the intensity profile for a given peak intensity $\rho_{0}$, one might resort to direct numerical techniques [e.g., treating Eq. (B2a) as a boundary-value problem and applying the shooting method (see Fig. 11)]. Alternatively, Eq. (B4) can be transformed into an integral equation. In the domain $\xi>0$, where $d \rho_{\mathrm{b}} / d \xi<0$, it can be shown that $\rho_{\mathrm{b}}$ may be obtained from

$$
\begin{aligned}
\frac{\rho_{\mathrm{b}}(\xi)}{\rho_{0}}=\exp [ & -2 \sqrt{2} \rho_{\mathrm{sat}} \int_{0}^{\xi} d X\left\{\frac{1}{\rho_{\mathrm{b}}(X)} \ln \left[1+\frac{\rho_{\mathrm{b}}(X)}{\rho_{\mathrm{sat}}}\right]\right. \\
& \left.\left.-\frac{1}{\rho_{0}} \ln \left(1+\frac{\rho_{0}}{\rho_{\mathrm{sat}}}\right)\right\}^{1 / 2}\right],
\end{aligned}
$$

which may be solved iteratively (e.g., in tandem with the non-degenerate bistability condition) [25].

After implementing the coordinate discussed in Sec. V, one can write down the more general frequency-shifted bright soliton solution of Eq. (B1), namely

$$
\begin{aligned}
u_{\mathrm{b}}(\tau, \zeta)= & \rho_{0}^{1 / 2} \exp \left[-\operatorname{sgn}\left(\Theta_{\mathrm{b}}\right) \sqrt{2} \rho_{\mathrm{sat}} \int_{0}^{\Theta_{\mathrm{b}}(\tau, \zeta)} d X\left\{\frac{1}{\rho(X)} \ln \left[1+\frac{\rho(X)}{\rho_{\mathrm{sat}}}\right]-\frac{1}{\rho_{0}} \ln \left(1+\frac{\rho_{0}}{\rho_{\mathrm{sat}}}\right)\right\}^{1 / 2}\right] \\
& \times \exp \left[i \Omega \tau \pm i \sqrt{1+4 \kappa \beta_{\mathrm{b}}-4 \kappa \Omega\left(\alpha+\frac{\Omega}{2}\right)} \frac{\zeta}{2 \kappa}\right] \exp \left(-i \frac{\zeta}{2 \kappa}\right)
\end{aligned}
$$

where $\Theta_{\mathrm{b}}(\tau, \zeta)$ and $W_{\mathrm{b}}$ are given by Eqs. (26) and (25b), respectively. We note in passing, but do not rigorously prove here, that the double-doping saturable nonlinearity proposed by Gatz and Herrmann [27] can be treated in exactly the same way and their solution (expressed in terms of an integral equation) generalized to spatiotemporal regimes accordingly. Since bright soliton (B6) is now known, it is now instructive to consider its stability properties in relation to those of solution (27a).

\section{Soliton stability}

For completeness, we thus consider briefly the same class of perturbed bright soliton initial-value problem for Eq. (B1) as in Sec. VII. The input pulse $u_{\mathrm{b}}(\tau, 0)$ is defined by solution (B6) but where the dilation factor $\left(1+2 \kappa W_{\mathrm{b}}^{2}\right)^{1 / 2}$ is omitted from the upper limit [i.e., from $\left.\Theta_{\mathrm{b}}(\tau, 0)\right]$. For straightforward comparison with earlier simulations, the same frequency shifts are retained to control the strength of the perturbation $(\Omega=4,8,12$, and 16) and peak intensities $\rho_{0}=2.298$ and $\rho_{0}=8.763$ are used (though these values do not necessarily lie on the bistability curve [25]). We also note that for fixed peak intensity and saturation parameters, the two-level model tends to have a stronger nonlinear response than the phenomenological model of Wood et al. [28].

Comparing Figs. 12(a) and 6(a) shows that quantitatively similar reshaping curves can be expected for the low-intensity waves. More pronounced differences can appear for higher-intensity waves, where the long-term limit cycle-type oscillations associated with reshaping solitons of Eq. (1) may be absent from the predictions of model

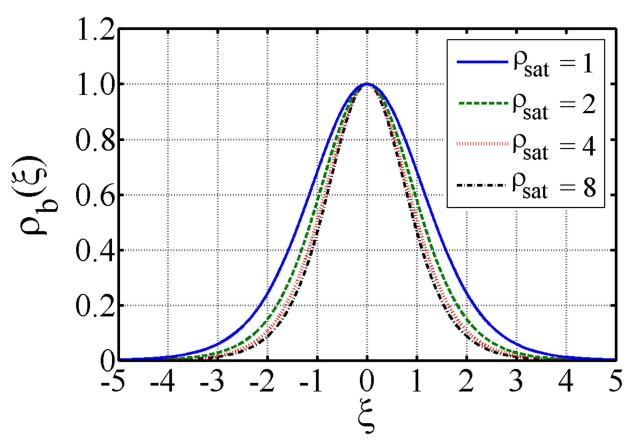

FIG. 11: (color online) Bright soliton intensity distributions obtained by solving Eq. (B2a) numerically for $\rho_{0}=1.0$ (they are universal since they are insensitive to variations in $\kappa, \alpha$, and $\left.V_{0 \mathrm{~b}}\right)$. Differences between the stationary solutions of Eqs. (1) and (B1) occur predominantly at low values of $\rho_{\text {sat }}$ (c.f. Fig. 1). 

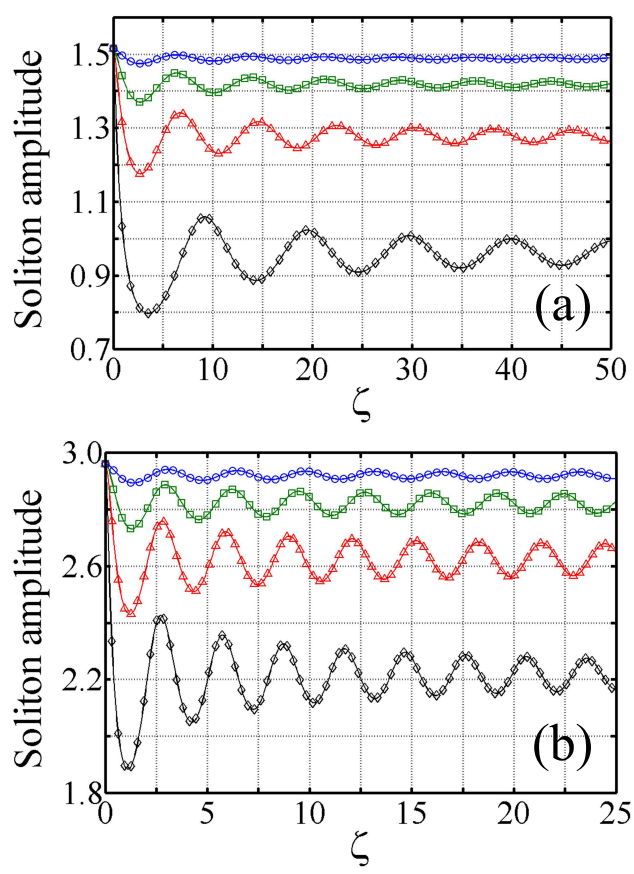

FIG. 12: (color online) Evolution of the bright soliton peak amplitude when the initial waveform for Eq. (B1) has a peak amplitude of (a) $\rho_{0}=2.298$ and (b) $\rho_{0}=8.763$. System parameters: $\rho_{\mathrm{sat}}=4.0, s=+1, \alpha=1.0, \kappa=1.0 \times 10^{-3}$. Blue circle: $\Omega=4$. Green square: $\Omega=8$. Red triangle: $\Omega=12$. Black diamond: $\Omega=16$.

(B1) [compare Figs. 12(b) and 6(b)]. One finds, instead, oscillatory features (with variations on a shorter longitudinal scalelength) that vanish as $\zeta \rightarrow \infty$ to leave a stationary solution. The simulations presented here and in Sec. VII B thus demonstrate that two different spatiotemporal saturable-nonlinearity models possess soliton solutions with similar stability characteristics.

\section{Appendix C: Modulational instability}

\section{Perturbation dispersion relation}

Here, we analyze the MI characteristics for a fullysecond-order envelope equation with a generic nonlinearity function $f\left(|u|^{2}\right)$,

$$
\kappa \frac{\partial^{2} u}{\partial \zeta^{2}}+i\left(\frac{\partial u}{\partial \zeta}+\alpha \frac{\partial u}{\partial \tau}\right)+\frac{s}{2} \frac{\partial^{2} u}{\partial \tau^{2}}+f\left(|u|^{2}\right) u=0
$$

where $f(0)=0$. Typical examples of $f$ include cubicquintic systems such as $f\left(|u|^{2}\right)=\gamma_{2}|u|^{2}+\gamma_{4}|u|^{4}$ [12], and saturable systems that may have either a simple form, $f\left(|u|^{2}\right)=|u|^{2}\left(1+|u|^{2} / \rho_{\text {sat }}\right)^{-1}[$ c.f., Eq. (B1)], or one that is more complicated,

$$
f\left(|u|^{2}\right)=\frac{1}{2} \frac{2+|u|^{2} / \rho_{\text {sat }}}{\left(1+|u|^{2} / \rho_{\text {sat }}\right)^{2}}|u|^{2}
$$

[c.f., Eq. (1)]. The two cw families are described by

$$
u_{\mathrm{cw}}(\tau, \zeta)=\rho_{0}^{1 / 2} \exp \left( \pm i \sqrt{1+4 \kappa \beta_{\mathrm{cw}}} \frac{\zeta}{2 \kappa}\right) \exp \left(-i \frac{\zeta}{2 \kappa}\right),
$$

where $\rho_{0} \equiv\left|u_{\mathrm{cw}}\right|^{2}$ is the uniform intensity, $\beta_{\mathrm{cw}} \equiv f\left(\rho_{0}\right)$, and the \pm sign determines the longitudinal sense of propagation ( + for forwards in $\zeta,-$ for backwards).

Perturbed solutions are now sought that have the form

$$
\begin{aligned}
u(\tau, \zeta)= & \rho_{0}^{1 / 2}[1+\epsilon a(\tau, \zeta)] \exp \left( \pm i \sqrt{1+4 \kappa \beta_{\mathrm{cw}}} \frac{\zeta}{2 \kappa}\right) \\
& \times \exp \left(-i \frac{\zeta}{2 \kappa}\right),
\end{aligned}
$$

where $\epsilon \ll \mathcal{O}(1)$ is a small parameter and $a(\tau, \zeta)$ is a complex function with $\mathcal{O}(1)$ magnitude describing perturbations to both the amplitude and phase of the underlying $\mathrm{cw}$ solution. It thus follows that, within a linear approximation, $|u(\tau, \zeta)|^{2} \equiv \rho \simeq \rho_{0}+\epsilon \rho_{0}\left(a+a^{*}\right) \equiv \rho_{0}+\Delta \rho(\tau, \zeta)$. The nonlinearity function $f$ can then be Taylor-expanded around the cw intensity $\rho_{0}$ so that

$$
f\left(|u|^{2}\right) \simeq f\left(\rho_{0}+\Delta \rho\right) \simeq \beta_{\mathrm{cw}}+\epsilon \rho_{0} f^{\prime}\left(\rho_{0}\right)\left(a+a^{*}\right),
$$

where $f^{\prime}\left(\rho_{0}\right) \equiv d f(\rho) /\left.d \rho\right|_{\rho=\rho_{0}}$ parametrizes the leadingorder correction [e.g., $f^{\prime}\left(\rho_{0}\right)=\gamma_{2}+2 \gamma_{4} \rho_{0}$ for a cubicquintic nonlinearity, or $f^{\prime}\left(\rho_{0}\right)=\left(1+\rho_{0} / \rho_{\text {sat }}\right)^{-2}$ and $f^{\prime}\left(\rho_{0}\right)=\left(1+\rho_{0} / \rho_{\text {sat }}\right)^{-3}$ for the two saturation models, respectively]. Higher-order terms in the expansion, such as $(\Delta \rho)^{2}$ and $(\Delta \rho)^{3}$ etc., are neglected in linear analysis.

Substitution of Eqs. (C3a) and (C3b) into Eq. (C1a) shows that $a$ must satisfy the linearized equation at $\mathcal{O}(\epsilon)$,

$$
\begin{aligned}
\kappa \frac{\partial^{2} a}{\partial \zeta^{2}} \pm i \sqrt{1+4 \kappa \beta_{\mathrm{cw}}} \frac{\partial a}{\partial \zeta}+ & i \alpha \frac{\partial a}{\partial \tau}+\frac{s}{2} \frac{\partial^{2} a}{\partial \tau^{2}} \\
& +\rho_{0} f^{\prime}\left(\rho_{0}\right)\left(a+a^{*}\right) \simeq 0 .
\end{aligned}
$$

Following the method detailed in Ref. [11], the Fourier modes of Eq. (C4) have a complex propagation constant $K_{\mathrm{p}}$ (allowing for potential growth of the perturbation wave) and frequency $\Omega_{\mathrm{p}}$ that are connected by the quartic dispersion relation

$$
\begin{aligned}
& \kappa^{2} K_{\mathrm{p}}^{4}-\left[1+4 \kappa \beta_{\mathrm{cw}}+2 \kappa \rho_{0} f^{\prime}\left(\rho_{0}\right)-s \kappa \Omega_{\mathrm{p}}^{2}\right] K_{\mathrm{p}}^{2} \\
& \mp 2 \alpha \Omega_{\mathrm{p}} \sqrt{1+4 \kappa \beta_{\mathrm{cw}}} K_{\mathrm{p}} \\
& \quad+\left(\frac{1}{2} \Omega_{\mathrm{p}}^{2}\right)\left[\left(\frac{1}{2} \Omega_{\mathrm{p}}^{2}\right)-2 \alpha^{2}-2 s \rho_{0} f^{\prime}\left(\rho_{0}\right)\right]=0 .
\end{aligned}
$$

While the four roots of Eq. (C5) can be written down exactly [51], they are algebraically cumbersome and do not provide much further physical insight. However, one regime of key importance of that corresponding to longwavelength excitations. 


\section{Long-wavelength instabilities}

Neglecting all $\kappa$-dependent contributions to the MI spectrum can be expected to recover the long-wavelength result from conventional pulse theory. By considering a forward-travelling host wave, perturbation dispersion relation (C5) is well-described by the quadratic approximation

$$
K_{\mathrm{p}}^{2}+2 \alpha \Omega_{\mathrm{p}} K-\left(\frac{1}{2} \Omega_{\mathrm{p}}^{2}\right)\left[\left(\frac{1}{2} \Omega_{\mathrm{p}}^{2}\right)-2 \alpha^{2}-2 s \rho_{0} f^{\prime}\left(\rho_{0}\right)\right] \simeq 0
$$

which has the two solution branches

$$
K_{\mathrm{p}}=-\alpha \Omega_{\mathrm{p}} \pm \sqrt{\left(\frac{1}{2} \Omega_{\mathrm{p}}^{2}\right)\left[\left(\frac{1}{2} \Omega_{\mathrm{p}}^{2}\right)-2 s \rho_{0} f^{\prime}\left(\rho_{0}\right)\right]} .
$$

The term at $-\alpha \Omega_{\mathrm{p}}$ is transformed away after a Galilean boost to the local time-frame, leaving the familiar result for MI in NLS-based models. One can thus deduce that the long-wavelength MI properties of the system are essentially independent of $\kappa$, and that they occur whenever $2 s \rho_{0} f^{\prime}\left(\rho_{0}\right)>\Omega_{\mathrm{p}}^{2} / 2$.

Our principal interest lies with model $(\mathrm{C} 1 \mathrm{~b})$, where $f^{\prime}\left(\rho_{0}\right) \geq 0$ for $\rho_{0} \geq 0$. In the anomalous-GVD regime (where $s=+1$ ), it follows that MI appears in the longwave band $0<\Omega_{\mathrm{p}}^{2}<4 \rho_{0} f^{\prime}\left(\rho_{0}\right)$. The most unstable frequency $\Omega_{\mathrm{p} 0}$ is then easily calculated from $\Omega_{\mathrm{p} 0}^{2}=$ $2 \rho_{0} f^{\prime}\left(\rho_{0}\right)$. In contrast, Eqs. (C6a) and (C6b) predict that there is no long-wavelength $\mathrm{MI}$ in the normal-GVD regime (where $s=-1$ ). Hence, the background wave of dark soliton (28a) is expected to be always stable against small background disturbances.
[1] T. Dauxois and M. Peyrard, Physics of Solitons (Cambridge University Press, Cambridge, 2006).

R. K. Dodd, J. C. Eilbeck, J. D. Gibbon, and H. C. Morris, Solitons and Nonlinear Wave Equations (Academic Press, London, 1982).

G. L. Lamb, Elements of Soliton Theory (John Wiley and Sons, New York, 1980).

[2] Y. S. Kivshar and B. A. Malomed, Rev. Mod. Phys. 61, 763 (1989).

V. G. Makhankov, Phys. Rep. 35, 1 (1978)

A. C. Scott, F. Y. F. Chu, and D. W. McLaughlin, Proc. IEEE 61, 1443 (1973).

[3] V. E. Zakharov and A. B. Shabat, Sov. Phys. JETP 34, 62 (1972).

V. E. Zakharov and A. B. Shabat, Sov. Phys. JETP 37, 823 (1973).

[4] A. Hasegawa and F. Tappert, Appl. Phys. Lett. 23, 142 (1972).

A. Hasegawa and F. Tappert, Appl. Phys. Lett. 23, 171 (1972).

L. F. Mellenauer, R. H. Stolen, and J. P. Gordon, Phys. Rev. Lett. 45, 1095 (1980).

A. M. Weiner, J. P. Heritage, R. J. Hawkins, R. N. Thurston, and E. M. Kirschner, Phys. Rev. Lett. 61, 2445 (1988).

P. Emplit, J. P. Hamaide, F. Reynaud, C. Frohly, and A. Barthelemy, Opt. Commun. 62, 374 (1987).

[5] Y. S. Kivshar, Opt. Quantum Electron. 30, 571 (1998). Y. S. Kivshar and B. Luther-Davies, Phys. Rep. 298, 81 (1998).

[6] J. M. Christian, G. S. McDonald, T. F. Hodgkinson, and P. Chamorro-Posada, Phys. Rev. Lett. 108, 034101 (2012).

[7] J. M. Christian, G. S. McDonald, and P. ChamorroPosada, Phys. Rev. A 76, 033833 (2007).

J. M. Christian, G. S. McDonald, and P. ChamorroPosada, Phys. Rev. A 81, 053831 (2010).

[8] A. B. Aceves, O. V. Shtyrina, A. M. Rubenchik, M. P. Fedoruk, and S. K. Turitsyn, Phys. Rev. A 91, 033810 (2015).

B. A. Malomed, D. Mihalache, F. Wise, and L. Torner, J. Opt. B: Quantum Semiclass. Opt. 7, R53 (2005).
F. Wise and P. Di Trapani, Optics \& Photonics News, February 7, 2002.

S. Raghavan and G. P. Agrawal, Opt. Commun. 180, 377 (2000).

X. Liu, L. Qian, and F. Wise, Phys. Rev. Lett. 82, 4631 (1999).

S.-S. Yu, C.-H. Chien, Y. Lai, and J. Wang, Opt. Commun. 119, 167 (1995).

[9] J. Burguete, H. Chaté and F. Daviaud, N. Mukolobwiez, Phys. Rev. Lett. 82, 3252 (1999).

M. van Hecke, Phys. Rev. Lett. 80, 1896 (1998).

I. Aranson, H. Levine, and L. Tsimring, Phys. Rev. Lett. 72, 2561 (1994).

H. Chaté, Nonlinearity 7, 185 (1994).

K. Nozaki and N. Bekki, Phys. Rev. Lett. 51, 2171 (1983).

[10] F. Biancalana and C. Creatore, Opt. Express. 16, 14822 (2008).

V. M. Agranovich and V. L Ginzburg, Crystal Optics with Spatial Dispersion, and Excitons (Springer, Berlin, 1984).

[11] J. M. Christian, G. S. McDonald, T. F. Hodgkinson, and P. Chamorro-Posada, Phys. Rev. A 86, 023838 (2012). J. M. Christian, G. S. McDonald, T. F. Hodgkinson, and P. Chamorro-Posada, Phys. Rev. A 86, 023839 (2012).

[12] J. M. Christian, G. S. McDonald, and A. Kotsampaseris, companion article submitted to Physical Review A (2018).

[13] J. D. Jackson, Classical Electrodynamics, 3rd ed. (John Wiley and Sons, New York, 1999).

H. Goldstein, Classical Mechanics, 2nd ed. (AddisonWesley, Philippines, 1980).

[14] P. Mandel, Theoretical Problems in Cavity Nonlinear Optics (Cambridge University Press, New York, 1997).

[15] M. Karlsson, Phys. Rev. A 46, 2726 (1992).

[16] R. W. Boyd, Nonlinear Optics, 2nd ed. (Academic Press, San Diego, 2003).

[17] K. I. Pushkarov and D. I. Pushkarov, Rep. Math. Phys. 17, 37 (1980).

K. I. Pushkarov, D. I. Pushkarov, and I. V. Tomov, Opt. Quantum Electron. 11, 471 (1979).

[18] Y. Chen, Phys. Rev. E 55, 1221 (1997). 
[19] Y. R. Shen, The Principles of Nonlinear Optics, (John Wiley and Sons, California, 2003).

[20] P. Roussignol, D. Ricard, J. Lukasik, and C. Flytzanis, J. Opt. Soc. Am. B 4, 5 (1987).

J.-L. Coutaz and M. Kull, J. Opt. Soc. Am. B 8, 95 (1991).

[21] T. Catunda and L. A. Cury, J. Opt. Soc. Am. B 7, 1445 (1990).

[22] Q. Wang Song, X. Wang, R. R. Birge, J. D. Downie, D. Timucin, and C. Gary, J. Opt. Soc. Am. B 15, 1602 (1998).

[23] L. Demenicis, A. S. L. Gomes, D. V. Petrov, C. B. de Araújo, C. P. de Melo, C. G. dos Santos, and R. SoutoMaior, J. Opt. Soc. Am. B 14, 609 (1997).

[24] S. Bian, J. Frejlich, and K. H. Ringhofer, Phys. Rev. Lett. 78, 4035 (1997).

D. N. Christodoulides and M. I. Carcalho, J. Opt. Soc. Am. B 12, 1628 (1995).

[25] S. Gatz and J. Herrmann, J. Opt. Soc. Am. B 14, 1795 (1997).

J. Herrmann, J. Opt. Soc. Am. B 8, 1507 (1991).

S. Gatz and J. Herrmann, J. Opt. Soc. Am. B 8, 2296 (1991).

[26] Y. Chen, Phys. Rev. A 45, 5215 (1992).

[27] S. Gatz and J. Herrmann, Opt. Lett. 17, 484 (1992).

[28] V. E. Wood, E. D. Evans, and R. P. Kenan, Opt. Commun. 69, 156 (1988).

[29] W. Krolikowski and B. Luther-Davies, Opt. Lett. 17, 1414 (1992).

[30] W. Krolikowski and B. Luther-Davies, Opt. Lett. 18, 188 (1993).

[31] S. Blair, Chaos, 10, 570 (2000).

K. Marinov, D. I. Pushkarov, and A. Shivarova, in Soliton-Driven Photonics, edited by A. D. Boardman and A. P. Sukhorukov (Kluwer Academic Press, Netherlands, 2001) p. 95.

C. Chen and S. Chi, Opt. Commun. 157, 170 (1998).

[32] R. Fedele, H. Schamel, and P. K. Shukla, Phys. Scr. T98, 18 (2002).

R. Fedele and H. Schamel, Eur. Phys. J. B 27, 313 (2002).

[33] W. Krolikowski, N. Akhmediev, and B. Luther-Davies, Phys. Rev. E 48, 3980 (1993).

[34] S. Gatz and J. Herrmann, IEEE J. Quantum Electron. 28, 1732 (1992).

[35] J. Herrmann, Opt. Commun. 91, 337 (1992).

[36] A. E. Kaplan, Phys. Rev. Lett. 55, 1291 (1985),

[37] I. S. Gradshteyn and I. M. Ryzhik, Tables of Integrals, Series, and Products, 7th ed. (Academic Press, New York, 2007).

[38] D. V Skryabin, J. Opt. Soc. Am. B 19, 529 (2002).

D. V. Skryabin, Phys. Rev. E 64, 055601 (2001).

D. E. Pelinovsky, V. V. Afanasjev, and Y. S. Kivshar,
Phys. Rev. A 53, 1940 (1996).

[39] I. V. Barashenkov, Phys. Rev. Lett. 77, 1193 (1996).

[40] D. E. Pelinovsky, Y. S. Kivshar, and V. V. Afanasjev, Phys. Rev. E 54, 2015 (1996).

[41] Y. S. Kivshar and V. V. Afansasjev, Opt. Lett. 21, 1135 (1996).

[42] V. V. Afanasjev, Y. S. Kivshar, C. R. Menyu, Opt. Lett. 21, 1975 (1996).

Y. S. Kivshar, Opt. Lett. 16, 892 (1991).

Y. S. Kivshar, Phys. Rev. A 43, 1677 (1991).

[43] M. Piché, J. F. Cormier, X. Zhu, Opt. Lett. 21, 845 (1996).

M. Karlsson and A. Höök, Opt. Commun. 104, 303 (1994).

[44] A. Mahalingam and K. Porsezian, Phys. Rev. E 64, 046608 (2001).

[45] Y. S. Kivshar and B. A. Malomed, Opt. Lett. 18, 485 (1993)

Y. S. Kivshar and V. V. Afanasjev, Opt. Lett. 16, 285 (1991)

Y. S. Kivshar, Phys. Rev. A 42, 1757 (1990).

[46] M. J. Ablowitz and D. E. Baldwin, Phys. Rev. E 86, 036305 (2012).

C. S. Gardner, J. M Greene, M. D. Kruskal, and R. M. Miura, Phys. Rev. Lett. 19, 1095 (1967).

N. J. Zabusky and M. D. Kruskal, Phys. Rev. Lett. 15, 240 (1965).

[47] M. Li and T. Xu, Phys. Rev. E 91, 033202 (2015).

J. A. Besley, P. D. Miller, and N. N. Akhmediev, Phys. Rev. E 61, 7121 (2000).

[48] J. K. Jang, M. Erkintalo, S. G. Murdoch, and S. Coen, Nat. Photon. 7, 657 (2013).

T. Yu, E. A. Golovchenko, A. N. Pilipetskii, and C. R. Menyuk, Opt. Lett. 22, 983 (1997).

Y. Kodama and K. Nozaki, Opt. Lett. 12, 1038 (1987). S. Wabnitz, Y. Kodama, and A. B. Aceves, Opt. Fib. Technol. 1, 187 (1995).

[49] A. Couairon, E. Gaizauskas, D. Faccio, A. Dubietis, and P. Di Trapani, Phys. Rev. E 73, 016608 (2006).

Y. Kominis, N. Moshonas, P. Papagiannis, K. Hizanidis, and D. N. Christodoulides, J. Opt. Soc. Am. B 30, 2924 (2005).

C. Conti, Phys. Rev. E 68, 016606 (2003).

C. Conti, S. Trillo, P. Di Trapani, G. Valiulis, A. Piskarskas, O. Jedrkiewicz, and J. Trull, Phys. Rev. Lett. 90, 170406 (2003).

[50] K. J. Blow and N. J. Doran, in Nonlinear Waves in Solid State Physics, NATO ADI Series B: Physics, Vol. 247, edited by A. D. Boardman, M. Bertolotti, and T. Twardowski (Plenum Press, New York, 1990) p. 325.

[51] M. Abramowitz and I. A. Stegun, Handbook of Mathematical Functions with Formulas, Graphs, and Mathematical Tables, (Dover, New York, 1972). 